\title{
Preliminary Studies of a Chromaticity Tracker
}

\author{
Cheng-Yang Tan \\ Accelerator Division/Tevatron
}

\begin{abstract}
A chromaticity tracker based on a method by D. McGinnis is proposed. This method starts with the slow modulation of the accelerating RF which causes the beam to respond to it. This beam modulation can be detected transversely with a Schottky pickup which after phase demodulation, the chromaticity can be calculated from it. However, to perform phase demodulation, the carrier frequency which is the betatron tune needs to be identified. The identification of the carrier frequency falls naturally onto the phase locked loop tune tracker which when locked to the betatron tune outputs this value in real time.
\end{abstract}




\section{INTRODUCTION}

Tracking the chromaticity especially during snapback is critical for the Large Hadron Collider (LHC). Various methods have been proposed to do this: the Bruening method, the head-tail method etc. In this paper, we will discuss the method by D. McGinnis. ${ }^{1}$ His idea is to phase modulate the beam with the accelerating RF and then detect this phase modulation with a transverse pickup. By phase demodulating this transverse signal, the chromaticity can be obtained. Although he had demonstrated that the chromaticity can be measured, he faced the problem that the betatron tune would drift over time and the phase demodulation circuit (which is a vector signal analyzer used in phase demodulation mode) would lose lock and thus would be unable to report the chromaticity. So, this was abandoned in favour of the head-tail method for measuring chromaticity operationally. ${ }^{2}$

Once the tune tracker (TT) was declared operational, it became clear that the McGinnis method could be made to work in synergy with the TT. ${ }^{3}$ First, the betatron tune drift problem would be solved because the TT would always be locked to the betatron tune (or to a synchrotron sideband of the betatron tune). Second the phase modulation would be chosen so that it would be outside the closed loop bandwidth of the TT and thus the TT would not "see" the modulation and respond to it. This choice of modulation frequency is important because it distinguishes it from the usual RF frequency change method where the TT would track the tune whenever the RF frequency $\omega_{\mathrm{RF}}$ is changed, i.e.

$$
\Delta Q_{0}=\chi \frac{\Delta p}{p}=-\frac{\chi}{\eta} \frac{\Delta \omega_{\mathrm{RF}}}{\omega_{\mathrm{RF}}}
$$

where $\Delta \omega_{\mathrm{RF}}$ is the RF frequency change, $\eta$ is the slip factor, $\Delta p / p$ is the relative momentum spread and $\Delta Q_{0}$ is the change in betatron tune from the nominal tune when $\Delta \omega_{\mathrm{RF}}=0$. Unfortunately, this usual method performed poorly whether done by hand or with the TT when coalesced beam is used because it yielded rather inaccurate chromaticities whenever octupoles were turned on to stabilize the beam. ${ }^{4}$ 
And so, we propose that the McGinnis method be used for the chromaticity tracker (CT) in the Tevatron and hopefully the results here will be useful for the LHC. In the next sections, we will go into the theory behind the McGinnis method and other topics that he had not discussed. We will calculate the parameters necessary for the CT to work without causing damage to the beam and show measurements which verify the McGinnis' method. Finally, we will show a possible implementation of the CT. In the Appendices, we will discuss the $3 \mathrm{D}^{-B B B Q^{5}}$ (Direct Diode Detection Baseband Tune) which is used at the LHC for their TT and a very narrow band filter which we propose to use in our CT. 
Theory 


\section{THEORY}

The McGinnis method ${ }^{1}$ starts with the modulation of the RF frequency $\dot{\phi}_{\mathrm{RF}}(t)$ with a sinusoid

$$
\dot{\phi}_{\mathrm{RF}}(t)=\omega_{\mathrm{RF}}+\Delta \phi_{\mathrm{mod}} \times \Omega_{\mathrm{mod}} \cos \left(\Omega_{\mathrm{mod}} t\right)
$$

where $\omega_{\mathrm{RF}}$ is the RF frequency when there is no modulation, $\Delta \phi_{\bmod }$ is the amplitude of the phase modulation and $\Omega_{\text {mod }}$ is the RF phase modulation. He showed that the betatron phase $\phi_{\beta}$ will then be modulated and its amplitude is linearly dependent on the chromaticity $\chi$. The relationship between $\phi_{\beta}$ to $\chi$ was calculated by him to be

$$
\phi_{\beta}(t)=\omega_{\mathrm{rev}} Q_{0} t+\frac{\Delta \phi_{\mathrm{mod}}}{h}\left(Q_{0}-\frac{\chi}{\eta}\right) \sin \Omega_{\bmod } t+\Delta \phi_{s}\left(Q_{0}-\frac{\chi}{\eta}\right) \sin \left(\Omega_{s} t+\theta_{s}\right)+\phi_{\beta_{0}}
$$

where $\omega_{\text {rev }}$ is the revolution frequency, $Q_{0}$ is the betatron tune, $h$ is the harmonic number, $\eta$ is the slip factor, $\Delta \phi_{s}$ is the amplitude of the synchrotron phase modulation, $\Omega_{s}$ is the synchrotron frequency, $\theta_{s}$ is the synchrotron phase and $\phi_{\beta_{0}}$ is the betatron phase at $t=0$.

In reality, we measure the betatron tune by taking the difference of the image current $I_{\Delta}$ between two plates i.e. at a pickup. This difference is

$$
\left.\begin{array}{rl}
I_{\Delta}(t)= & \omega_{\mathrm{rev}} q_{b} \frac{A}{\sqrt{\epsilon}} \cos \left[\phi_{\beta}(t)\right] \sum_{n=-\infty}^{\infty} \delta\left(\phi_{r}(t)-2 n \pi-\phi_{r}(0)\right) \\
= & \frac{\omega_{\mathrm{rev}} q_{b}}{2} \frac{A}{\sqrt{\epsilon}} \sum_{k=0}^{\infty} C_{k} \sum_{m=-\infty}^{\infty} \sum_{n=-\infty}^{\infty} J_{m}\left(Y_{+}\right) J_{n}\left(Z_{+}\right) \cos \left(\omega_{k m n}^{+} t+\psi_{+}\right) \\
& +\frac{\omega_{\mathrm{rev}} q_{b}}{2} \frac{A}{\sqrt{\epsilon}} \sum_{k=0}^{\infty} C_{k} \sum_{m=-\infty}^{\infty} \sum_{n=-\infty}^{\infty} J_{m}\left(Y_{-}\right) J_{n}\left(Z_{-}\right) \cos \left(\omega_{k m n}^{-} t+\psi_{-}\right)
\end{array}\right\}
$$

where

$$
\phi_{r}(t)=\omega_{\text {rev }} t+\frac{\Delta \phi_{\text {mod }}}{h} \sin \left(\Omega_{\text {mod }} t\right)+\Delta \phi_{s} \sin \left(\Omega_{s} t+\theta_{s}\right)
$$

and $q_{b}$ is the charge of the bunch, $A$ is the betatron amplitude at the pickup, $\epsilon$ is the transverse emittance, $C_{0}=1 / 2 \pi, C_{k}=1 / \pi, Y_{ \pm}=\left(k \pm Q_{0} \mp \frac{\chi}{\eta}\right) \Delta \phi_{s}, Z_{ \pm}=\left(k \pm Q_{0} \mp \frac{\chi}{\eta}\right) \frac{\Delta \phi_{\text {mod }}}{h}$, $\psi_{ \pm}=k \phi_{r}(0) \pm \phi_{\beta}(0)$, and $\omega_{k m n}^{ \pm}=\left(k \pm Q_{0}\right) \omega_{\mathrm{rev}}+m \Omega_{s}+n \Omega_{\mathrm{mod}}$ 
Therefore, (4) shows that at a given betatron mode $k$, there are an infinite number of modes $m$ associated with the synchrotron frequency. Around any mode $(k, m)$, there are an infinite number of modes $n$ associated with the RF modulation. See Figure 1. Note that these modes are not necessarily "good" because they can be degenerate, for example if $\Omega_{s}$ is an integer multiple of $\Omega_{\mathrm{mod}}$.

In general, the TT will just lock to one synchrotron sideband and so we need to show we can still obtain the chromaticity by phase demodulating this sideband by using it as the carrier frequency. Suppose the synchrotron line that the TT locks to is mode $(k, m,+)$, then its mode frequency is $\omega_{k m}^{+}=\left(k+Q_{0}\right) \omega_{\mathrm{rev}}+m \Omega_{s}$. The spectrum of modes associated with the RF modulation in temporal space is

$$
\left.\begin{array}{rl}
I_{\Delta}(t ; k, m,+) & \propto \sum_{n=-\infty}^{\infty} J_{n}\left(Z_{+}\right) \cos \left(\omega_{k m}^{+} t+n \Omega_{\text {mod }} t+\psi_{+}\right) \\
& =\cos \left(\omega_{k m}^{+} t+Z_{+} \sin \Omega_{\bmod } t+\psi_{+}\right)
\end{array}\right\}
$$

by using the well known formula

$$
e^{i Z \sin \theta}=\sum_{n=-\infty}^{\infty} J_{n}(Z) e^{i n \theta}
$$

This result shows that the carrier frequency is $\omega_{k m}^{+}$and the phase is modulated by $Z_{+} \sin \Omega_{\text {mod }} t$. More importantly, it also shows that we can phase demodulate using any synchrotron line as the carrier to obtain $\chi$ because the peak amplitude of the modulation $Z_{+}$is both independent of $m$ and the amplitude of the synchrotron line. A similar argument will show that this is also true for the synchrotron line $(k, m,-)$ where $Z_{-}$is the peak modulation amplitude.

\section{D-BBQ}

The LHC TT uses the 3D-BBQ pickup and we have analyzed it in Appendix I in order to understand how we can use it as a detector for the CT. From there, we have found that 
(a) Betatron "+" lines only

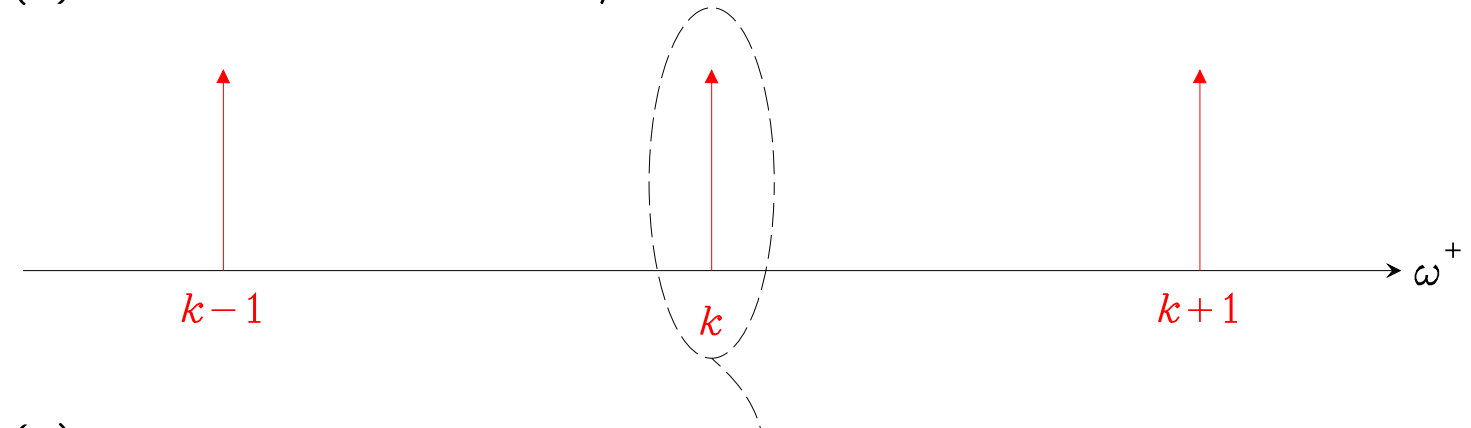

(b) Zoomed in betatron line and synchrotron lines

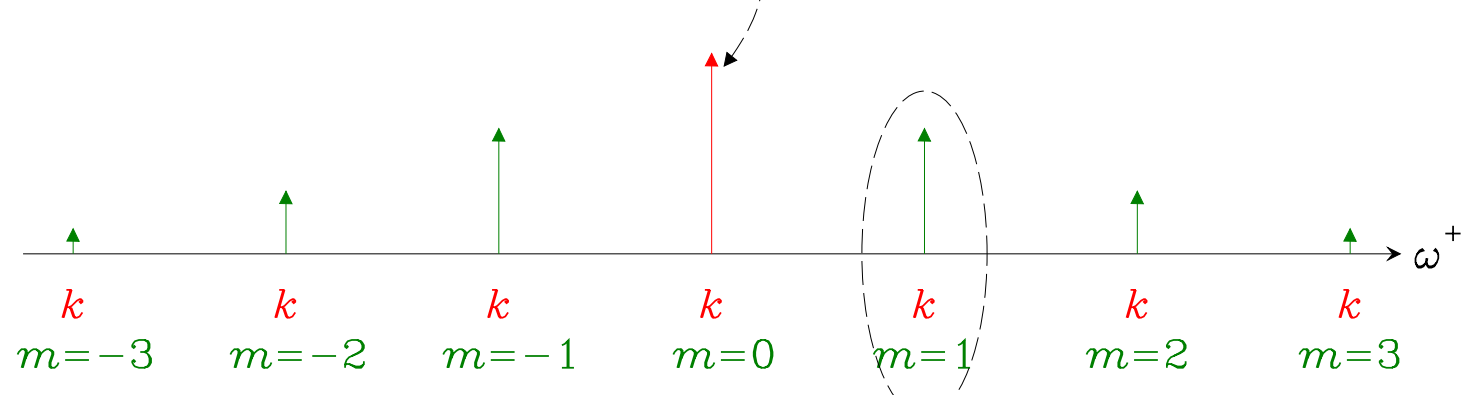

(c) Zoomed in synchrotron line and forcéd modulation lines

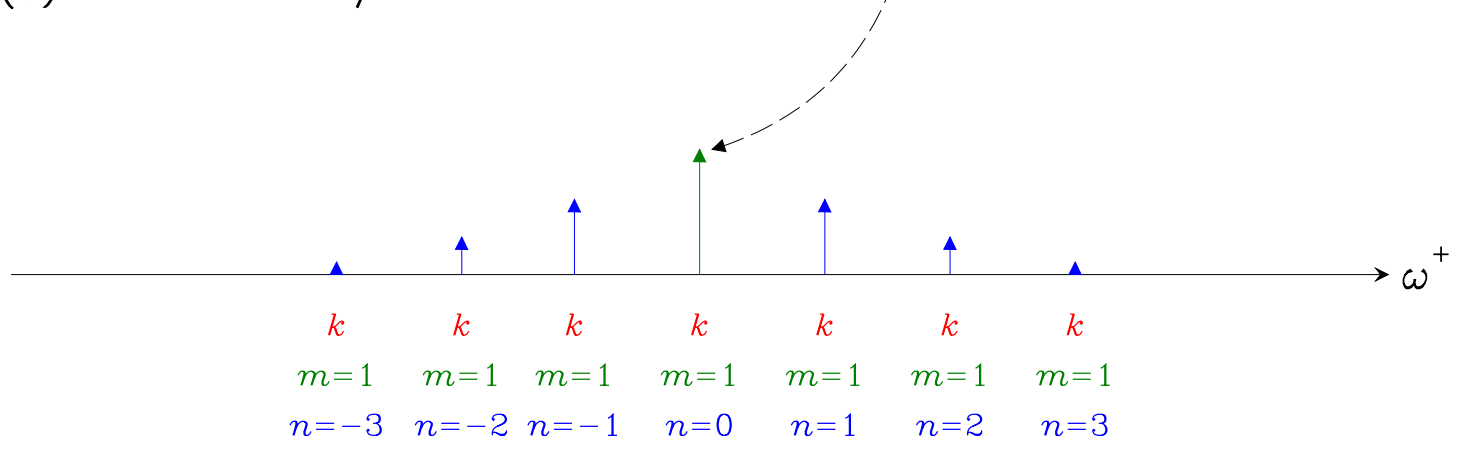

Figure 1 (a) The $\left(k+Q_{0}\right)$ betatron lines (b) The $(k,+)$ betatron line with its synchrotron lines (c) The $(k, m,+)$ synchrotron line and its forced modulation lines. Note that we have just drawn a representative cartoon here, because $k, m, n$ extends from $-\infty$ to $+\infty$.

the 3D-BBQ measures the tune which corresponds to the $k=0$ mode and thus the formula for calculating the chromaticity $\chi$ if the TT locks to the synchrotron line $(k=0, m,+)$ is

$$
\chi=-\eta\left[\frac{Z_{+} h}{\Delta \phi_{\mathrm{mod}}}-Q_{0}\right]
$$




\section{Example}

For the Tevatron, if we use the 21.4 MHz Schottky detector, we measure the betatron tune from the lower sideband of $k=449$. Since the Tevatron betatron tunes are between $0.5 \omega_{\mathrm{rev}}$ and $0.6 \omega_{\mathrm{rev}}$, this means that $k-Q_{0} \approx 448.5$. The peak of the phase demodulation $\left|Z_{-}\right|$is related to the chromaticity $\chi$ by the formula

$$
\begin{aligned}
|\chi| & =\eta\left|\frac{\sqrt{2}\left|Z_{-}\right| h}{\Delta \phi_{\bmod }}-\left(k-Q_{0}\right)\right| \\
& = \begin{cases}0.0029|9018.4| Z_{-}|-448.5| & \text { if } \Delta \phi_{\bmod }=10^{\circ} \\
0.0029|18036.8| Z_{-}|-448.5| & \text { if } \Delta \phi_{\bmod }=5^{\circ}\end{cases}
\end{aligned}
$$

if $\left|Z_{-}\right|$is measured in units of rad.rms (from a vector signal analyzer) $h=1113$, and $\eta=0.0029$.

If we use the 3D-BBQ as the detector, which in the Tevatron measures the $\left|Z_{+}\right|$peak, (8) is approximately

$$
\begin{aligned}
|\chi| & \approx\left|\frac{Z_{+} \sqrt{2} h \eta}{\Delta \phi_{\bmod }}\right| \\
& =52.30\left|Z_{+}\right| \quad \text { if } \Delta \phi_{\bmod }=5^{\circ}
\end{aligned}
$$

\section{Sign of the Chromaticity (Method I)}

It is obvious from the previous example that the sign of the chromaticity cannot be directly determined from just the height of $\left|Z_{ \pm}\right|$alone. The mode lines at $(k, m, 0 ;+)$, $(k, m, 1 ;+)$ and at $(k, m, 0 ;-),(k, m, 1 ;-)$ can be used to do this. The ratio of these heights is

$$
R\left(Z_{ \pm}\right)=\frac{J_{0}\left(Z_{ \pm}\right)^{2}}{J_{1}\left(Z_{ \pm}\right)^{2}}
$$

For the range of chromaticities $-20<\chi<20$ in the Tevatron, we can plot the upper and lower sidebands $k+Q_{0} \approx=449.5$ and $k-Q_{0} \approx 448.5$ using the same numbers as before. 


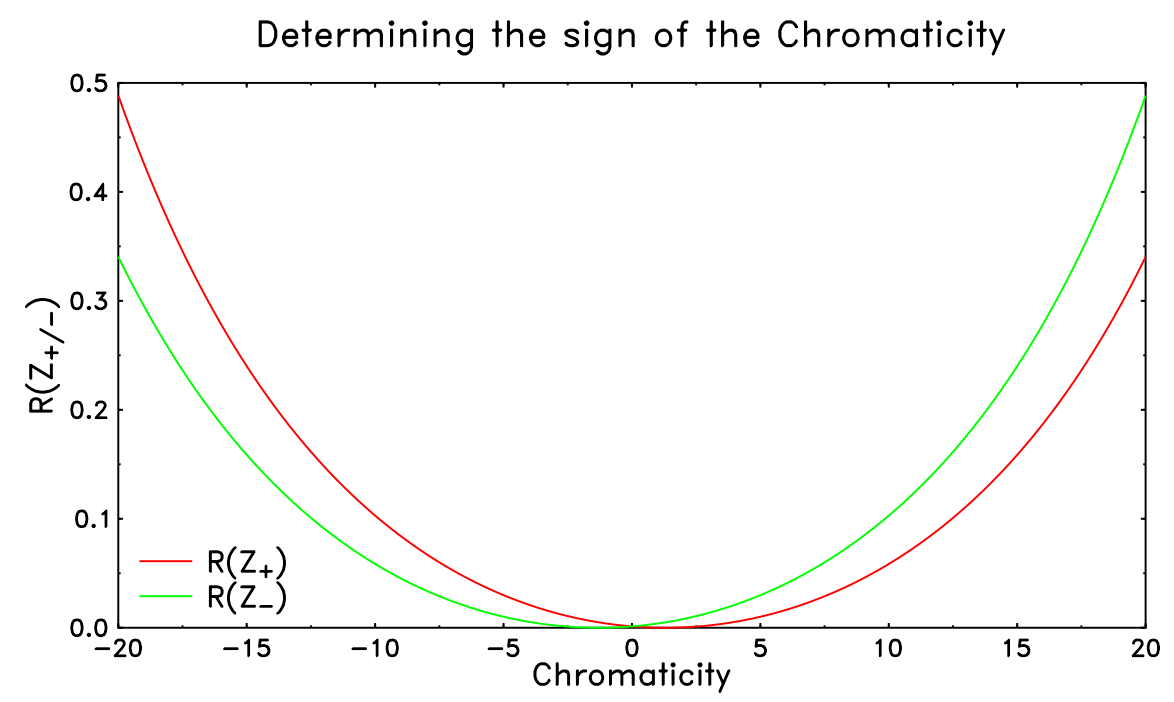

Figure 2 This graph shows how the sign of the chromaticity can be determined by using $R$. If $\chi>0$ then $R\left(Z_{-}\right)>R\left(Z_{+}\right)$.

Figure 2 shows that if $\chi>0$ then $R\left(Z_{-}\right)>R\left(Z_{+}\right)$and vice versa.

Sign of the Chromaticity (Method II)

Unfortunately, Method I is not compatible with the TT because TT only uses one sideband. This method requires that the modulation frequency be transmitted from the source which is close to the RF cavities to the location of the TT and CT. Once this modulation frequency is transmitted to the location of the CT, we can mix it with the demodulated signal from the CT, i.e.

$$
W_{ \pm}=\left(Z_{ \pm} \sin \Omega_{\bmod } t\right) \times B \sin \left(\Omega_{\bmod } t+\theta\right)
$$

where $B>0$ is the amplitude and $\theta$ is the phase of the modulation frequency. $\theta$ is measured at the CT location w.r.t. the modulation frequency at the source. Note that if $\Omega_{\bmod } \approx(2 \pi \times 23) \mathrm{s}^{-1}$, then its wavelength is approximately $10^{7} \mathrm{~m}$, this means that despite 
the fact that the modulation source and the $\mathrm{CT}$ are at two different locations, $\theta \approx 0$. Thus

$$
W_{ \pm}=\frac{B Z_{ \pm}}{2}\left(1-\cos 2 \Omega_{\bmod } t\right)
$$

We only want the DC term, $W_{\mathrm{DC}}=B Z_{ \pm} / 2$

$$
\frac{2 W_{\mathrm{DC}}}{B}=\left(k-Q_{0}+\frac{\chi}{\eta}\right) \frac{\Delta \phi_{\mathrm{mod}}}{h}
$$

if we consider only the $Z_{-}$term for the $21.4 \mathrm{MHz}$ Schottky. And to get the sign of the chromaticity, we need to do a little bit of processing to get

$$
\frac{2 W_{\mathrm{DC}}}{B}-\left(k-Q_{0}\right) \frac{\Delta \phi_{\mathrm{mod}}}{h}=\frac{\chi}{\eta} \times \frac{\Delta \phi_{\mathrm{mod}}}{h}
$$

Therefore, not only the sign of the chromaticity but the chromaticity itself can be obtained from here.

It would seem that we would have to pull a cable from the source of the modulation to the location of the TT. There is one way to overcome this: we can build two modulation sources but with each phase locked to the Tevatron RF, therefore there will be a fixed phase relationship between the two sources and so a cable pull becomes unnecessary. This technique will be used for the CT.

\section{Choice of $\Omega_{\bmod }$}

The choice of $\Omega_{\bmod }$ is at least obvious: $\Omega_{\bmod }$ should not be at the synchrotron frequency $\Omega_{s}$. The reason is that we do not want to excite the beam and blow up its emittance or cause beam loss. The motion of the beam with phase modulation has been completely solved analytically by Huang. ${ }^{6}$ However, we will do this numerically using the difference 
equations

$$
\begin{gathered}
\varphi_{n+1}=\varphi_{n}+2 \pi h \eta\left(\frac{\Delta p}{p}\right)_{n+1}+ \\
\Delta \phi_{\bmod }\left\{\sin \left[2 \pi \nu_{\bmod } \sum_{k=1}^{n+1}\left[1+\eta\left(\frac{\Delta p}{p}\right)_{k}\right]\right]-\right. \\
\left.\sin \left[2 \pi \nu_{\bmod } \sum_{k=1}^{n}\left[1+\left(\frac{\Delta p}{p}\right)_{k}\right]\right]\right\} \\
\left(\frac{\Delta p}{p}\right)_{n+1}=\left(\frac{\Delta p}{p}\right)_{n}+\frac{e V}{\beta^{2} E_{s}}\left(\sin \varphi_{n}-\sin \varphi_{s}\right)
\end{gathered}
$$

where $\nu_{\bmod }=\Omega_{\bmod } / \omega_{\mathrm{rev}}, \beta \approx 1$ is the relativistic beta, $e$ is the the electron charge, $V$ is the peak voltage of the RF, $E_{s}$ is the energy of the synchronous particle and $\varphi_{s}$ is the synchronous phase. The initial distribution and its projections used in the simulations are shown in Figure 3. With this distribution we simulate for $2 \mathrm{~s}$ with $50 \times 10^{3}$ particles for

\begin{tabular}{|c|c|c|c|}
\hline$\nu_{\bmod }$ & $\begin{array}{c}\text { Phase Dist. } \sigma \\
{\left[{ }^{\circ}\right]}\end{array}$ & $\begin{array}{l}\text { Momentum Dist. } \boldsymbol{\sigma} \\
{\left[\frac{\eta}{\nu_{s}}\left(\frac{\Delta p}{p}\right)\right]}\end{array}$ & Remarks \\
\hline 0 & 25.7 & 0.43 & initial distribution \\
\hline$\nu_{s}$ & 43.6 & 0.50 & dist. no longer gaussian \\
\hline$\nu_{s} / 3$ & 25.8 & 0.43 & no growth \\
\hline
\end{tabular}
$\Delta \phi_{\bmod }=10^{\circ}, \Omega_{\bmod }=\Omega_{s}$ and $\Omega_{\bmod }=\Omega_{s} / 3$. As expected, we find that if $\Omega_{\bmod }=\Omega_{s}$, the emittance gets diluted by filamentation. From our simulations, we found that if $\Omega_{\bmod }<$ $\Omega_{s} / 3$, the emittance does not change. See Table 1 and Figure 4 .

Another consideration is that we want $\Omega_{\bmod }$ to be outside the closed loop bandwidth of the TT because we do not want the TT to track the phase modulation. See Figure 5. As long as $\Omega_{\bmod }>(2 \pi \times 20) \mathrm{s}^{-1}$, this requirement is satisfied. See the section Computer Simulations with the TT. 
Initial Phase Space Distribution

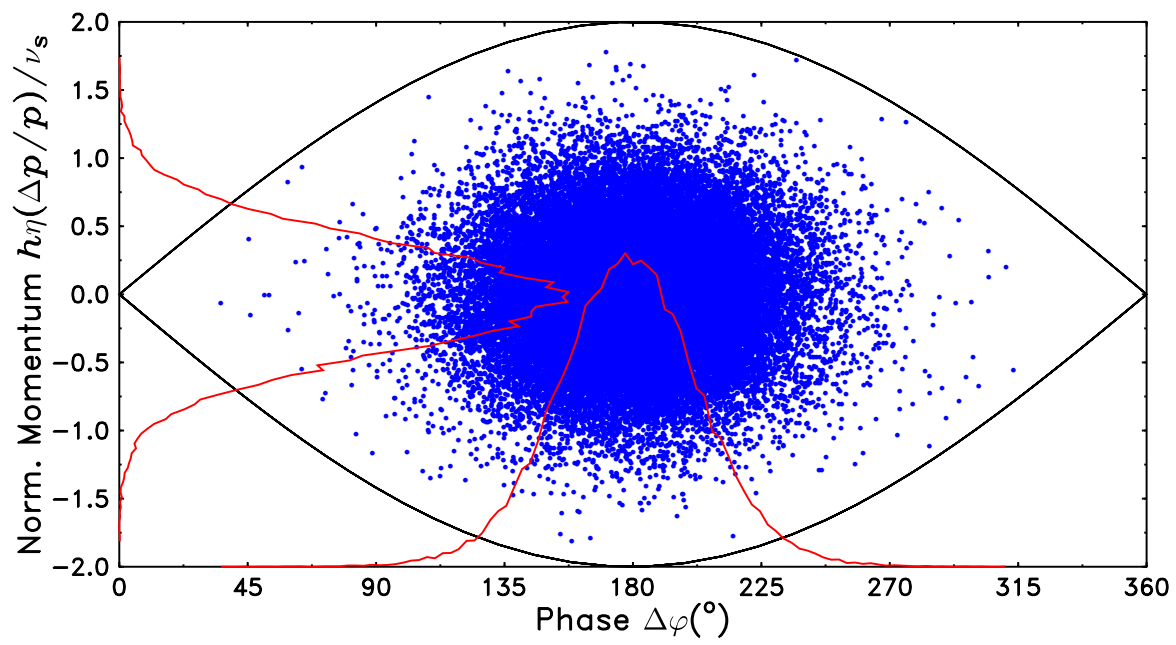

Figure 3 The initial distribution used in our simulation.

\section{Choice of $\Delta \phi_{\bmod }$}

There are two conditions which we have to consider for the choice of $\Delta \phi_{\text {mod }}$ :

(i) The maximum change in the tune $\dot{\phi}_{\beta}$ must be $<\left(0.1 \times 10^{-3}\right) \times \omega_{\text {rev }}$.

(ii) The bucket area, especially up the ramp is reduced with the modulation and this can be a problem.

The maximum change in tune due to the phase modulation comes from differentiating (3) w.r.t. time and only looking at the term $\delta \dot{\phi}_{\beta}$ which contains $\Omega_{\bmod }$

$$
\left|\frac{\delta \dot{\phi}_{\beta}}{\omega_{\mathrm{rev}}}\right|_{\max }=\left|\frac{\Delta \phi_{\mathrm{mod}} \times \Omega_{\mathrm{mod}}}{\omega_{\mathrm{RF}}}\left(Q_{0}-\frac{\chi}{\eta}\right)\right|<0.1 \times 10^{-3}
$$

For the typical parameters of the Tevatron, assuming that $\Omega_{\bmod }=(2 \pi \times 23) \mathrm{s}^{-1}, \omega_{\mathrm{RF}}=$ $2 \pi \times\left(53 \times 10^{6}\right) \mathrm{s}^{-1}, Q_{0}=(1-0.575), \chi=10$ and $\eta=0.0029$, we see that

$$
\Delta \phi_{\bmod }=0.07 \approx 4^{\circ}
$$



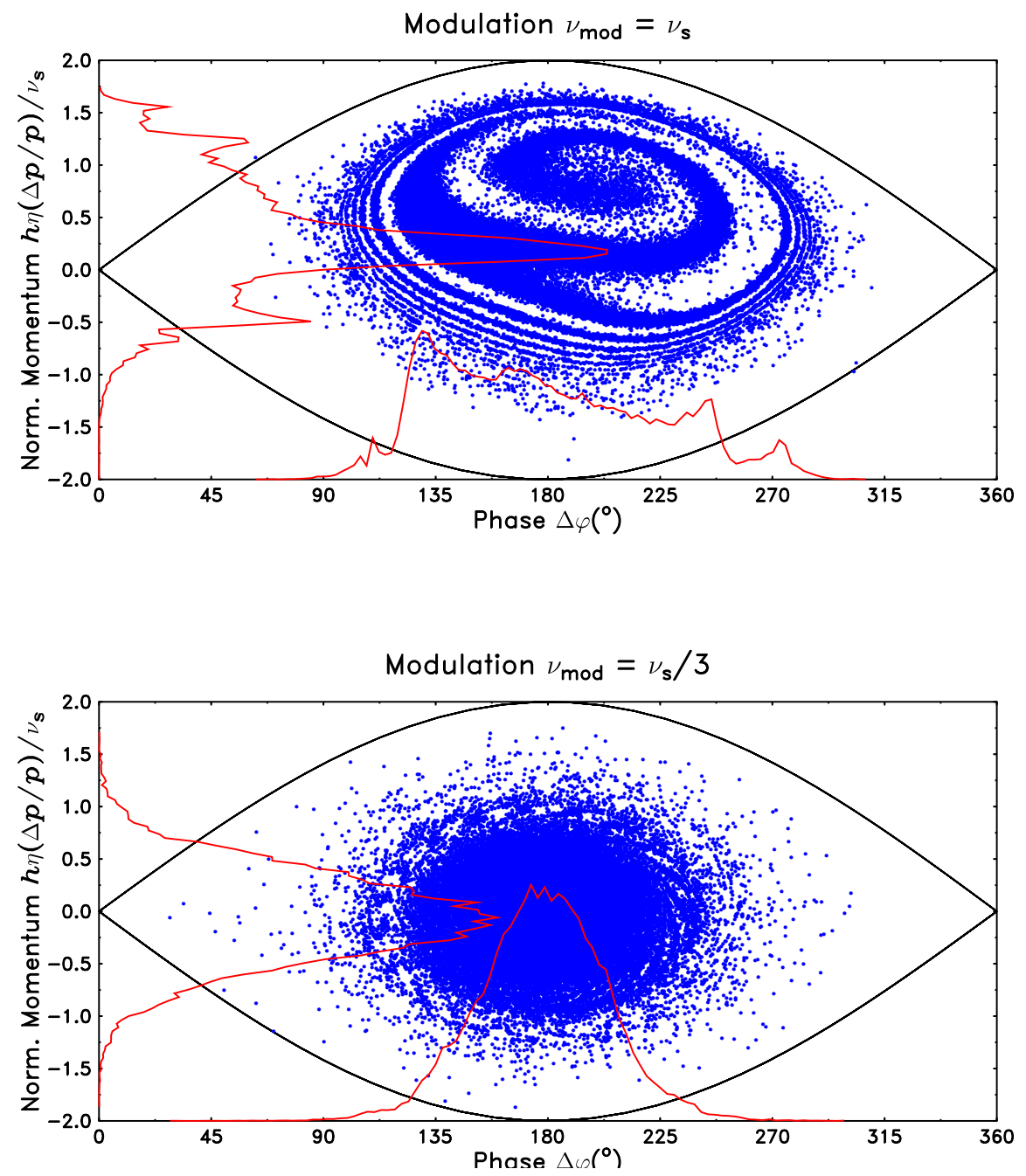

Figure 4 The computer simulation which shows that when $\nu_{\bmod }=$ $\nu_{s}$, the beam clearly dephases. For $\nu_{\bmod }=\nu_{s} / 3$, the emittance does not change.

\section{Bucket Area}

With the size of the phase modulation $\Delta \phi_{\text {mod }}$ determined to be about $4^{\circ}$, we will show that the bucket area is not affected by the phase modulation. 


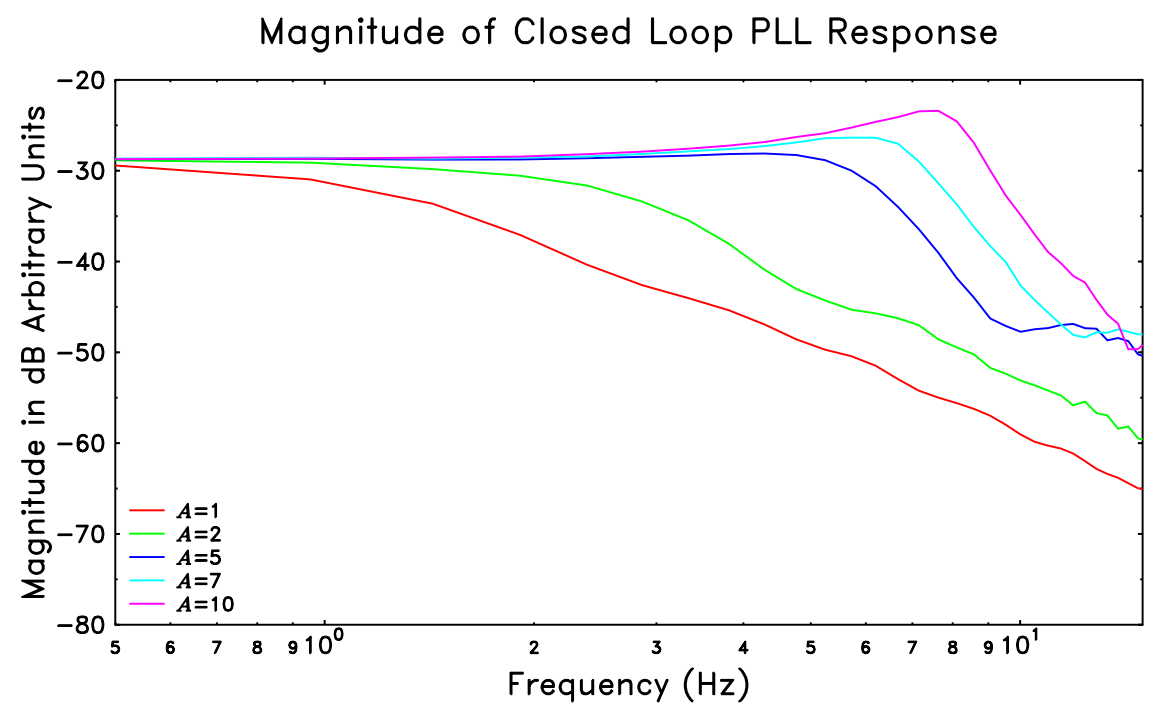

Figure 5 The measured closed loop response ${ }^{3}$ of the TT for various gain multipliers $A$. It is clear that as long as $A<10$, we want $\Omega_{\bmod }>$ $2 \pi \times 20 \mathrm{~s}^{-1}$.

The bucket area $\mathcal{A}$ is given approximately by ${ }^{7}$

$$
\mathcal{A}_{0} \approx \frac{16 \beta}{\omega_{\mathrm{RF}}} \sqrt{\frac{e V \cdot E_{s}}{2 \pi h|\eta|}} \times\left(\frac{1-\sin \varphi_{s}}{1+\sin \varphi_{s}}\right)
$$

If we let $\omega_{\mathrm{RF}} \rightarrow \dot{\phi}_{\mathrm{RF}}(t)$, then the relative change in bucket area $\Delta \mathcal{A} / \mathcal{A}_{0}$ because of the RF phase modulation is

$$
\begin{aligned}
\frac{\Delta \mathcal{A}}{\mathcal{A}_{0}} & =-\frac{\Omega_{\mathrm{mod}} \Delta \phi_{\mathrm{mod}}}{\dot{\phi}_{\mathrm{RF}}(t)} \cos \left(\Omega_{\mathrm{mod}} t\right) \\
& \approx-\frac{\Omega_{\mathrm{mod}} \Delta \phi_{\mathrm{mod}}}{\omega_{\mathrm{RF}}} \cos \left(\Omega_{\mathrm{mod}} t\right) \quad \text { if } \frac{\Delta \phi_{\mathrm{mod}} \Omega_{\mathrm{mod}}}{\omega_{\mathrm{RF}}} \ll 1 .
\end{aligned}
$$

The maximum reduction in the bucket area is when $\cos \Omega_{\bmod } t=1$, so

$$
\left(\frac{\Delta \mathcal{A}}{\mathcal{A}_{0}}\right)_{\max }=-\frac{\Delta \phi_{\bmod } \Omega_{\bmod }}{\omega_{\mathrm{RF}}}
$$

Putting in some numbers for the Tevatron and assuming that $\Omega_{\bmod }=(2 \pi \times 23) \mathrm{s}^{-1}$, $\omega_{\mathrm{RF}}=2 \pi \times\left(53 \times 10^{6}\right) \mathrm{s}^{-1}$ and $\Delta \phi_{\bmod }=4 \times \pi / 180$ then

$$
\left(\frac{\Delta \mathcal{A}}{\mathcal{A}_{0}}\right)_{\max }=-3 \times 10^{-8}
$$


which is really small. Thus there should not be any concern about the size of the bucket when the RF is modulated with the parameters which we intend to use.

\section{Radial Position Change}

For the Tevatron, the maximum dispersion is $D \sim 10 \mathrm{~m}$ and so the maximum change in radial position $\Delta r_{\max }$ due to the modulation is

$$
\begin{aligned}
\Delta r_{\max } & =\frac{D}{\eta} \times \frac{\Omega_{\mathrm{mod}} \Delta \phi_{\mathrm{mod}}}{\omega_{\mathrm{RF}}} \\
& =260 \mu \mathrm{m}
\end{aligned}
$$

by using the parameters of the previous section. Even with the helix open, there is still a few millimetres of physical aperture, thus the radial position change should not be a problem.

\section{Limitations}

There are two possible limitations in the McGinnis method. The first, from (3), shows that this method will fail at transition because $\eta=0$. The second is whether the longitudinal dampers will damp out the phase modulation. We can think of the slow phase modulation on the beam as a mode 0 coupled bunch mode. The Tevatron longitudinal

damper does not damp mode 0 at this time ${ }^{8}$, but instead relies on the RF frequency being set to the correct side of the revolution harmonic to keep the beam stable, i.e. using the Robinson stability criterion. Therefore, in principle, the longitudinal dampers should not affect this method.

Computer Simulations with the TT

We want to demonstrate with computer simulations that the TT will track the tune 
and not see the phase modulation if it is outside the TT's closed loop bandwidth. In these simulations, in order to make the oscillations visible, we have set $\Delta \phi_{\bmod }=20 \times \pi / 180$ and compare the results when $\Omega_{\bmod }=(2 \pi \times 1) \mathrm{s}^{-1}$ and $(2 \pi \times 23) \mathrm{s}^{-1}$. See Figure 6 . We deliberately placed a step in the betatron tune (blue trace) at $10 \mathrm{~s}$. For both cases, the TT tracks this step (red trace). However it is clear that for $\Omega_{\bmod }=(2 \pi \times 1) \mathrm{s}^{-1}$, the TT also tracks the modulation because the output of the phase detector (green trace) is close to zero. While for the $\Omega_{\bmod }=(2 \pi \times 23) \mathrm{s}^{-1}$ case, we can see from both the red and green traces that the TT clearly does not track. Therefore, from this simulation, we have confirmed that by setting the phase modulation frequency outside the closed loop bandwidth of the TT, the TT will ignore the phase modulation but still track the tune. Note: the maximum beam frequency change (blue trace) between the $\Omega_{\bmod }=(2 \pi \times 1) \mathrm{s}^{-1}$ and $(2 \pi \times 23) \mathrm{s}^{-1}$ comes from $(17)$

$$
\frac{\Omega_{\mathrm{mod}}=2 \pi \times 23}{\Omega_{\mathrm{mod}}=2 \pi \times 1}=23
$$

i.e. we expect and see that the maximum amplitude of the beam frequency oscillation from the $\Omega_{\bmod }=(2 \pi \times 23) \mathrm{s}^{-1}$ phase modulation is 23 times larger than the amplitude of the $\Omega_{\bmod }=(2 \pi \times 1) \mathrm{s}^{-1}$ phase modulation. 

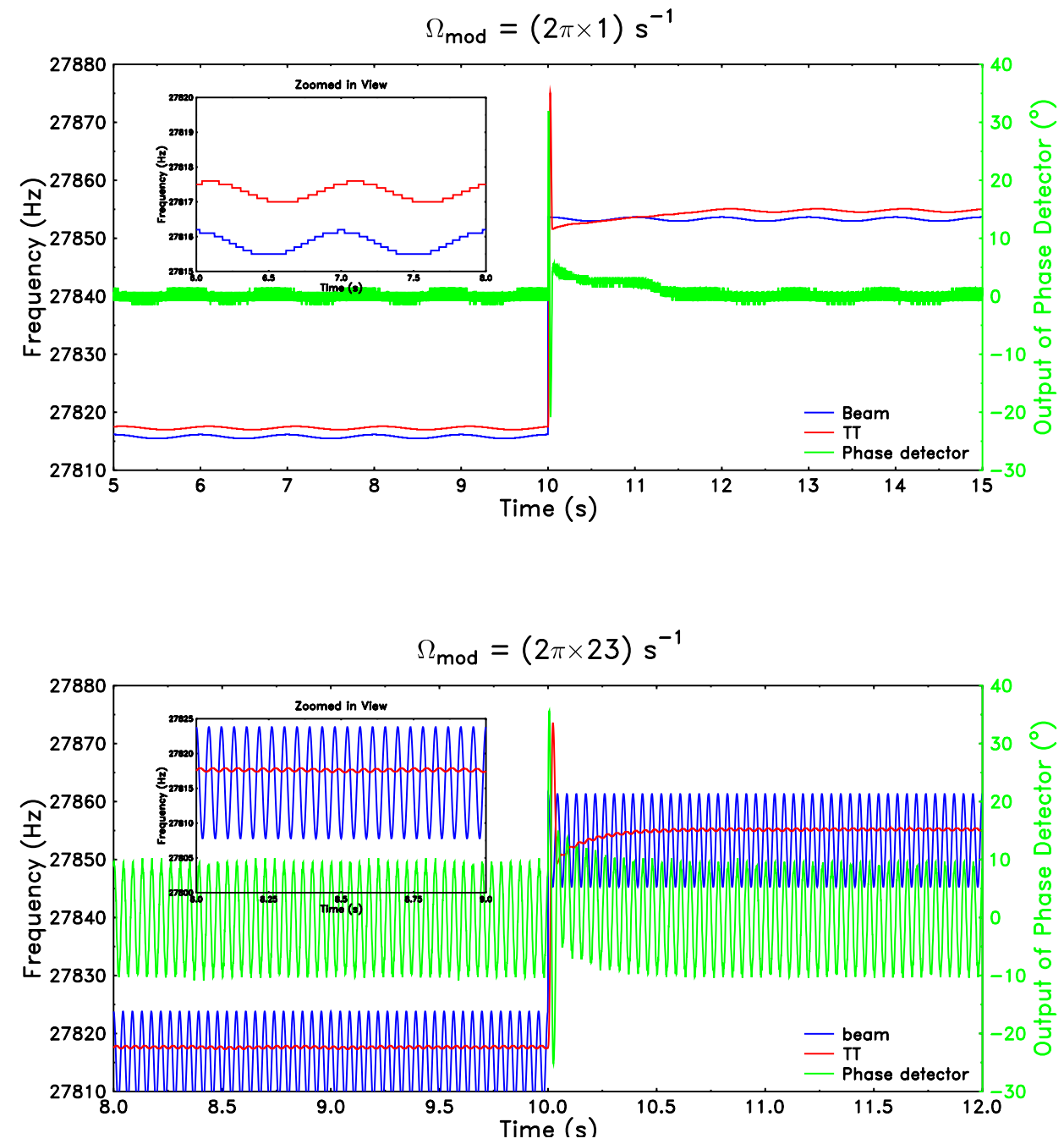

Figure 6 This simulation shows that for the same gain, when $\Omega_{\bmod }=(2 \pi \times 1) \mathrm{s}^{-1}$, the $\mathrm{TT}$ tracks the modulation and the $\mathrm{TT}$ is locked because the output of the phase detector is close to zero. However, when $\Omega_{\bmod }=(2 \pi \times 23) \mathrm{s}^{-1}$, the TT does not track the modulation but still tracks the step change in frequency. Here, the output of the phase detector is clearly not close to zero. 
Measurements 


\section{MEASUREMENTS}

All these measurements were performed by phase modulating the Tevatron RF at $\Omega_{\bmod }=(2 \pi \times 23) \mathrm{s}^{-1}$. The TT was used to lock to the betatron tune. Because the betatron tune was excited with a clear carrier from the TT, a vector signal analyzer (VSA) could then be used to demodulate the betatron tune. Unfortunately, for this setup we could not just type in the TT excitation frequency into the VSA and use zero span because the betatron tune drifts and so we must rely on the VSA's phase lock loop to find the carrier in a non-zero bandwidth. In these experiments, the VSA was set to a bandwidth of $200 \mathrm{~Hz}$ (and thus a $100 \mathrm{~Hz}$ span display of the phase demodulation) centred around the betatron tune. However, in this mode of operation, the VSA update rate was quite slow $\sim 2$ to 3 seconds because it needed steady state to be reached. The setup is shown in Figure 7.

Two sets of data were taken: $\Delta \phi_{\bmod }=10^{\circ}$ and $5^{\circ} .^{\dagger}$ The result from demodulating the betatron tune by the VSA is shown in Figure 8. Clearly, from here, the height of the $23 \mathrm{~Hz}$ peak for the $\Delta \phi_{\bmod }=10^{\circ}$ case is approximately twice that of the $\Delta \phi_{\bmod }=5^{\circ}$ case for the same chromaticity like we would expect from the $Z_{ \pm}$formula.

We varied the chromaticity $\chi$ and measured $\left|Z_{-}\right|$from the VSA. We then used the formula from (9) to to calculate $\chi$ from $\left|Z_{-}\right|$. The fit between the expected chromaticity $\chi_{\text {expected }}$ and the chromaticity from McGinnis' method $\chi_{\text {McGinnis }}$ for the two cases $\Delta \phi_{\bmod }=10^{\circ}$ and $5^{\circ}$ are shown in Figure 9 . The result of the fits were

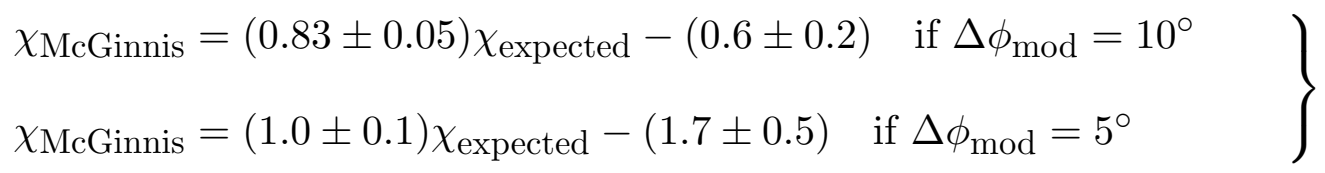

Note that the sign of the chromaticity, which cannot be determined directly from the

$\dagger$ The calibration of $\Delta \phi_{\bmod }$ is a factor of 2 larger than what we had expected. This will be investigated during the March to May 2006 shutdown. 

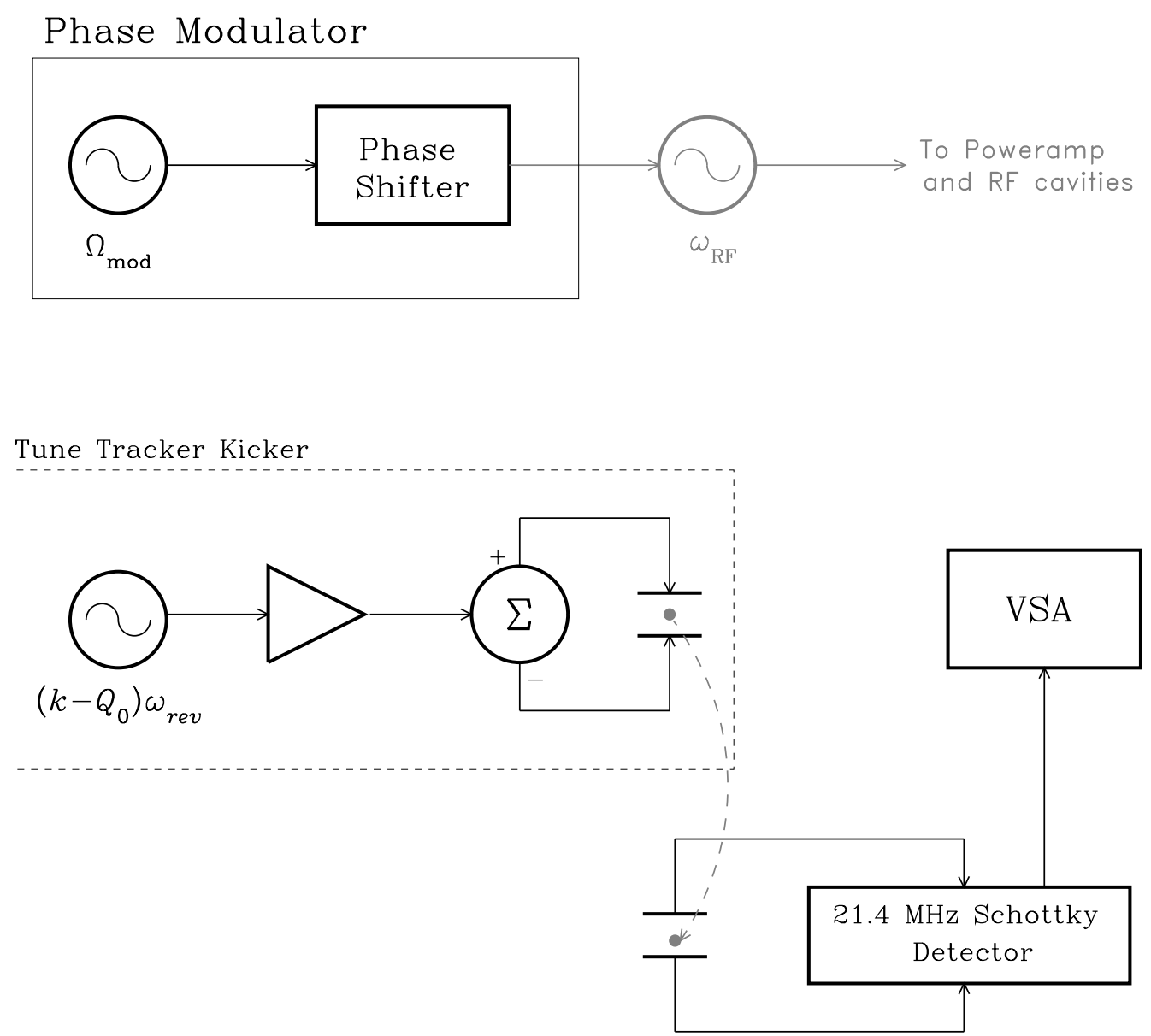

Figure 7 The setup for these measurements start with modulating the beam with the RF. The TT is locked to the betatron tune and its excitation frequency serves as the carrier for the VSA to phase demodulate the betatron tune.

height of the phase demodulation with the VSA, is assumed to be the same as that of $\chi_{\text {expected }}$

Note further that due to the unavailability of the head-tail method because of equipment failure, we were only able to measure chromaticity at one point with the RF change method (See Introduction) and then extrapolate chromaticity changes with a "calibrated" knob. And so, $\chi$ expected does have an error bar associated with it of \pm 0.5 units. 
Date: 01-14-06 Time: 03:17 AM

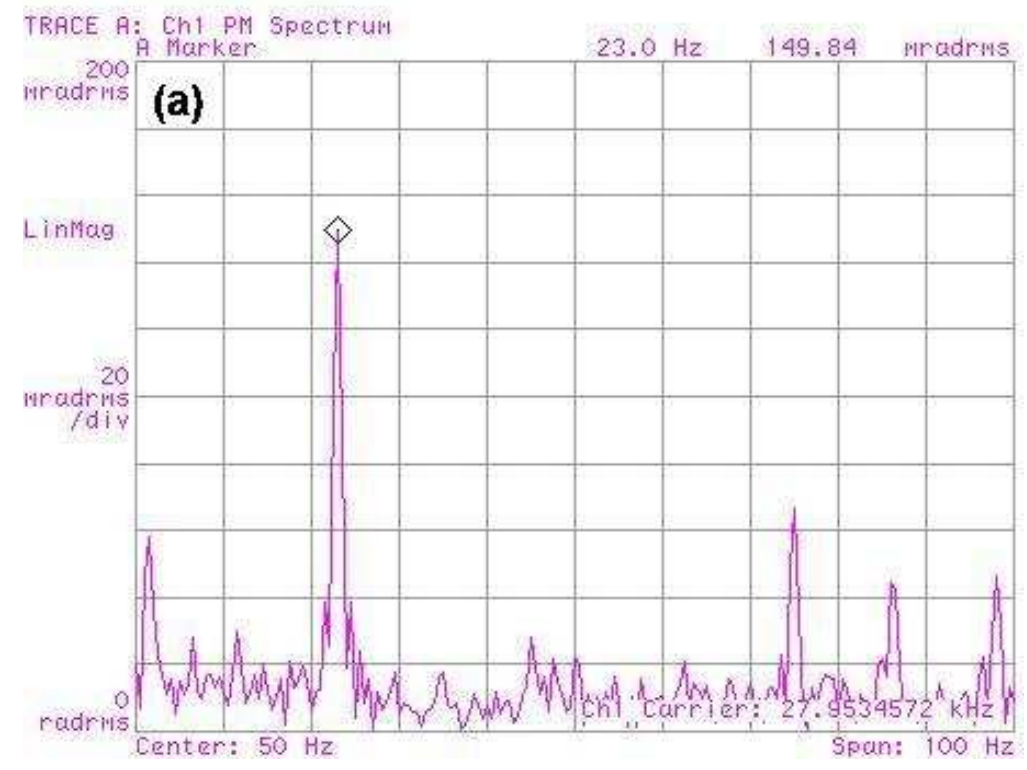

Date: 01-14-06 Time: 03:18 AM

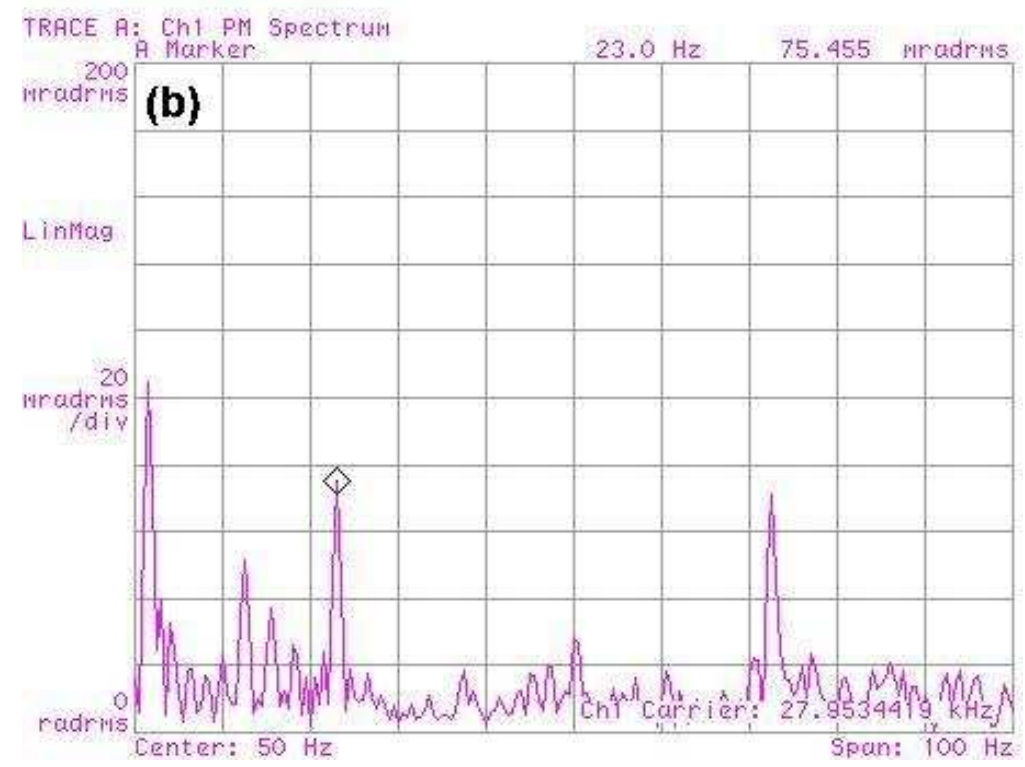

Figure 8 These figures show the result of demodulating the betatron tune. (a) shows the case when $\Delta \phi_{\bmod }=10^{\circ}$ and (b) shows the case when $\Delta \phi_{\text {mod }}=5^{\circ}$. Clearly the height of the $23 \mathrm{~Hz}$ peak of (a) is approximately twice that of (b) like we expect for the same chromaticity. 


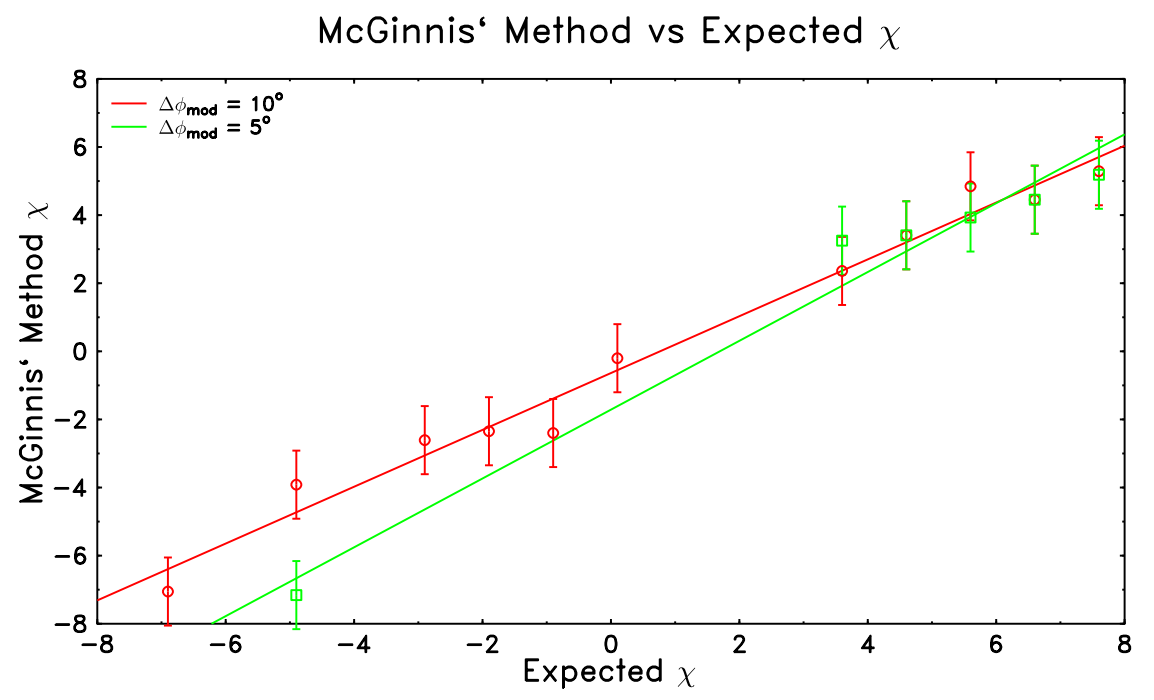

Figure 9 Comparing the McGinnis' method to the expected chromaticity for $\Delta \phi_{\bmod }=10^{\circ}$ and $5^{\circ}$.

\section{Other Measurements}

One of the things that we need to show is that the TT will ignore the phase modulation. From Figure 10, the phase modulation is turned on with $\Delta \phi_{\bmod }=5^{\circ}, \Omega_{\bmod }=(2 \pi \times 23) \mathrm{s}^{-1}$ and the TT is on and tracking. Clearly, we see that the TT tracks the tune changes when the chromaticity knob (the tune change is a side effect of changing the chromaticity with the chromaticity knob) is changed. As expected, the TT does not track the phase modulation because $\Delta \phi_{\text {mod }}$ is outside the closed loop bandwidth of the TT.

Obviously, there is a limit to the size of $\Delta \phi_{\text {mod }}$. When $\Delta \phi_{\bmod }=40^{\circ}$, the beam immediately falls out of the bucket (T:SBDPIS) while the total current T:BEAM remains constant after we turn on the modulation. For $\Delta \phi_{\text {mod }}=5^{\circ}$, the bunched beam current and the total current remains constant. This experiment shows that a $5^{\circ}$ modulation will not harm the beam. See Figures 10 and 11. The choice of $\Delta \phi_{\bmod }=5^{\circ}$ is also consistent with 


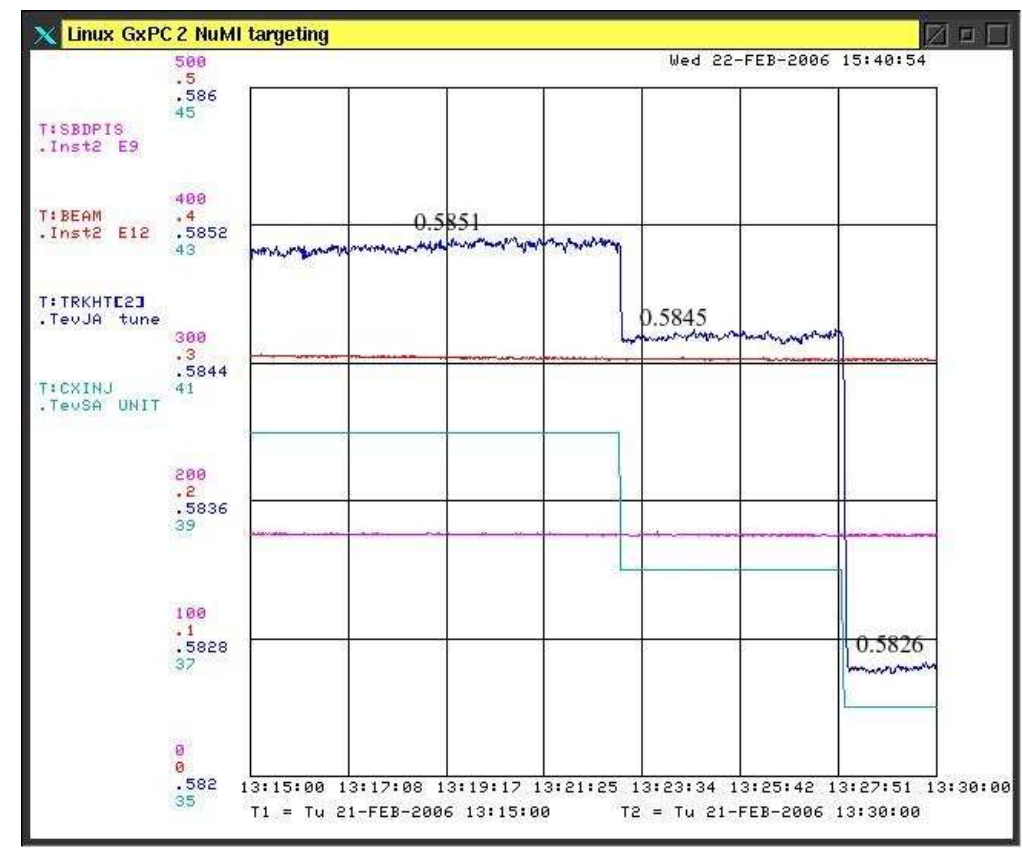

Figure 10 Tracking with $\Delta \phi_{\text {mod }}=5^{\circ}$. The tracked tune T:TRKHT[2] tracks the tune changes when the chromaticity knob is changed T:CXINJ. The bunched beam T:SBDPIS and the total current T:BEAM remains constant during this measurement.

the requirement that we do not want the betatron tune to move by more than $0.1 \times 10^{-3}$. See the Theory section.

\section{$3 \mathrm{D}-\mathrm{BBQ}$}

We did a study with the 3D-BBQ by replacing the $21.4 \mathrm{MHz}$ Schottky detector shown in Figure 7 with it. In this setup, the TT still works and tracks at $21.4 \mathrm{MHz}$. However, because of the periodicity of the beam at the pickup (See Appendix I), the 21.4 MHz kick from the TT is "down converted" and appears at baseband. The tune spectra measured by the 3D-BBQ with the TT turned on and phase modulation on/off is shown in Figure 12. It is clear from here that extra lines pop up because of the phase modulation. Figure 13 shows the result after phase demodulating a $\Delta \phi_{\text {mod }}=5^{\circ}$ modulation. In this measurement, we 


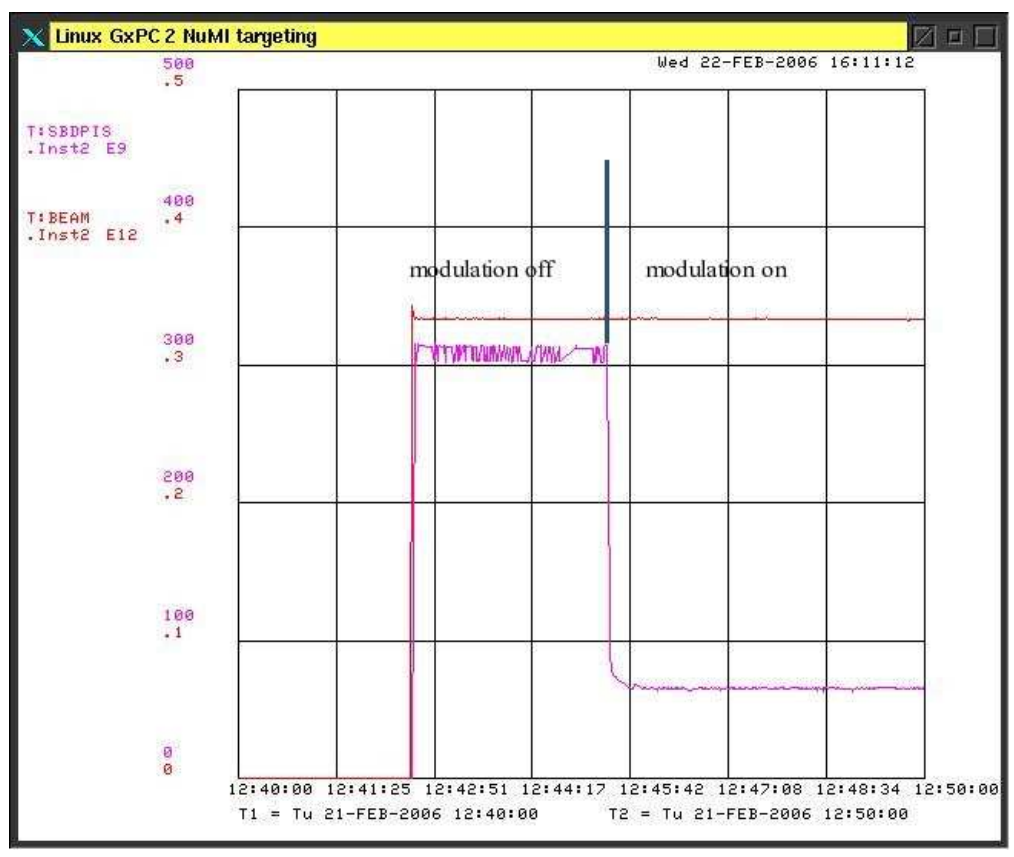

Figure 11 This shows that DC beam is created when $\Delta \phi_{\text {mod }}=$ $40^{\circ}$. The total current T:BEAM is constant while the bunched beam T:SBDPIS falls out of the bucket. Note that there is already DC beam in the Tevatron even before the modulation is turned on because $\mathrm{T}: \mathrm{SBDPIS} \neq \mathrm{T}: \mathrm{BEAM}$.

see that the phase demodulated spectra is much cleaner than that from the $21.4 \mathrm{MHz}$ Schottky of Figure 8.

For the chromaticity measurement, we were only able to measure three points for $\Delta \phi_{\text {mod }}=5^{\circ}$ because of time limitations. The results are shown in Table 2. (Note that in this measurement, $\chi_{\text {expected }}$ was also extrapolated from one chromaticity measurement with a "calibrated" knob.) The formula used for calculating $\chi_{\text {McGinnis }}$ comes from (10). Even from these three data points, we can see that the expected chromaticity and McGinnis method are close. A more complete and more careful study should show that the 3D-BBQ will work as a detector for the CT. 

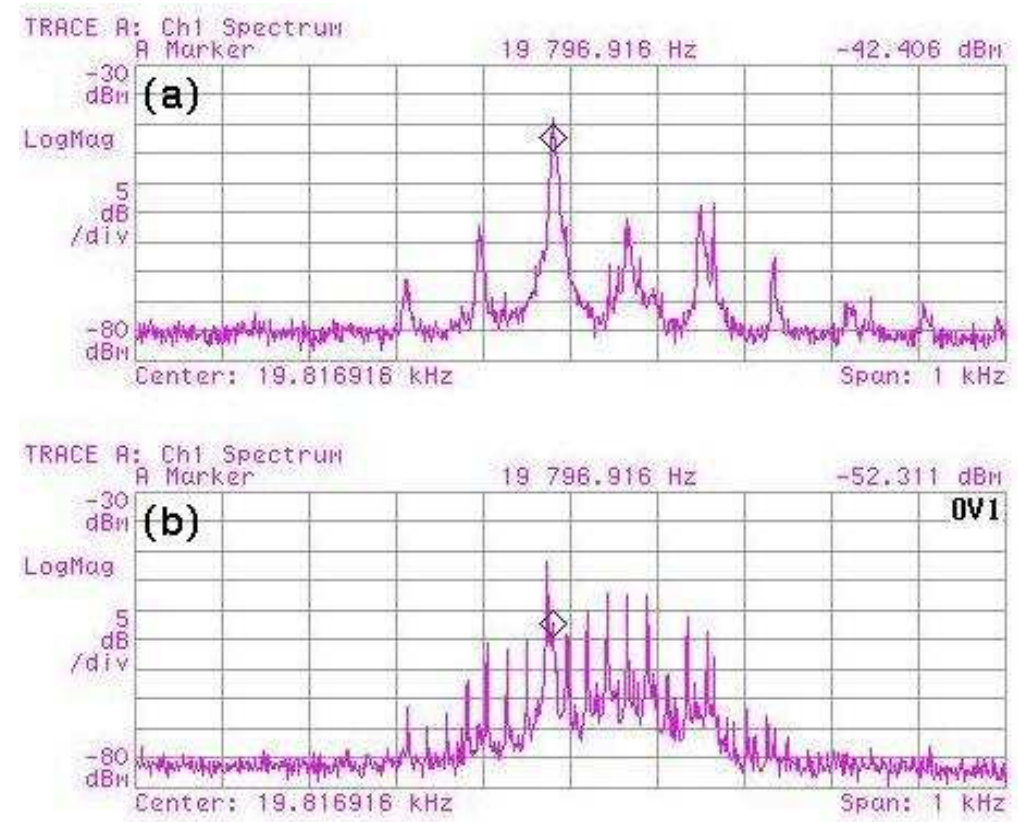

Figure 12 The tune spectra measured by the 3D-BBQ with the TT on in both (a) and (b). (b) shows the tune spectra with the phase modulation of $\Delta \phi_{\bmod }=40^{\circ}$ turned on.

\begin{tabular}{c|l}
\hline \multicolumn{2}{c}{ Table 2. Chromaticity Measured with 3D-BBQ } \\
\hline$\chi_{\text {expected }}$ & $\chi_{\text {McGinnis }}$ \\
$8^{*}$ & 11 \\
6 & 7 \\
4 & 4 \\
\hline \hline
\end{tabular}

"**" is the measured chromaticity. The other $\chi_{\text {expected }}$ values are extrapolated from a "calibrated" knob. 
Date: 03-25-04 Time: 05:25 AM

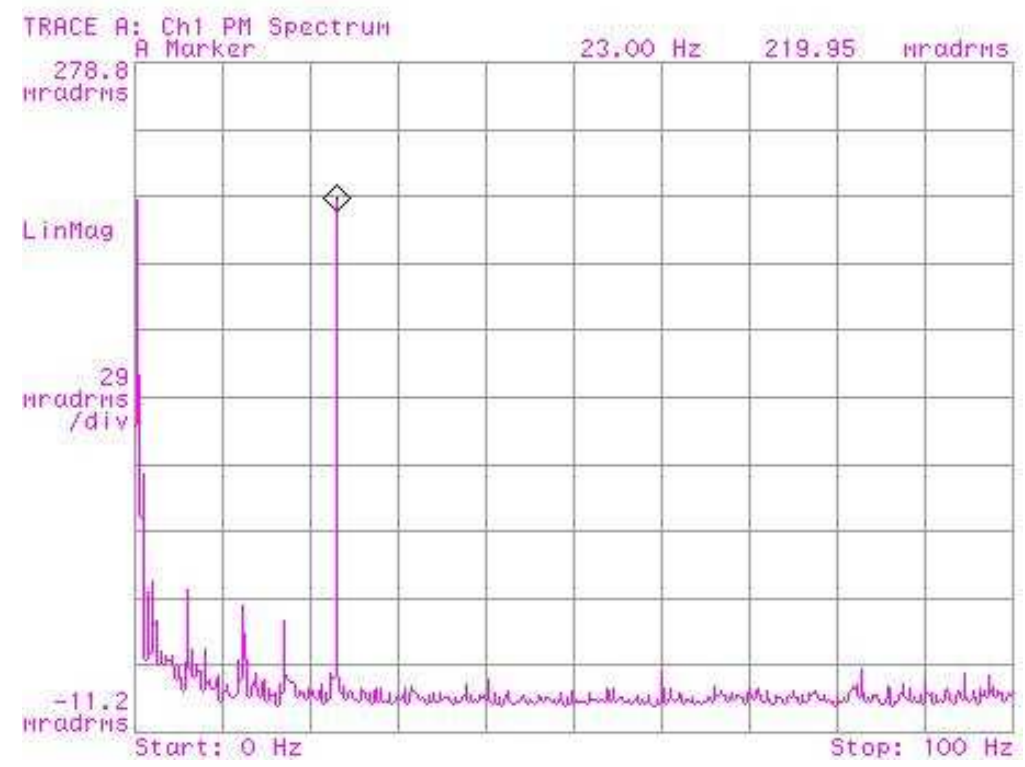

Figure 13 The demodulated spectrum from the 3D-BBQ for $\Delta \phi_{\bmod }=$ $5^{\circ}$. 


\section{Implementation}




\section{IMPLEMENTATION}

The simplest and most obvious implementation of the CT is to simply add a very narrow band filter like a Sliding Goertzel Algorithm (SGA) filter (See Appendix II) to the output of the phase detector in the TT circuit shown in Figure 14. ${ }^{\dagger}$ The actual implementation will not be the same as shown in this figure because of the limitations of the original TT design at Fermilab plus the necessity of building the sign evaluation circuit.

Our proposed implementation is shown in Figure 15. The components are:

(i) A hardware phase detector circuit.

(ii) The software SGA.

(iii) Two phase locked direct digital synthesiser (DDS) modules. One located at the low level RF room for generating the phase modulation. Another located at the TT which generates the reference modulation.

The schematics are being drawn up right now and are of course, subject to change as we refine the final design.

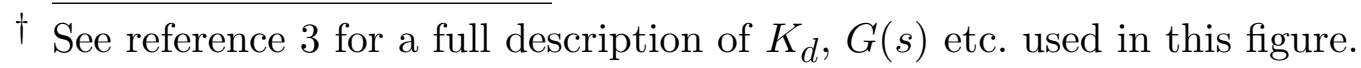




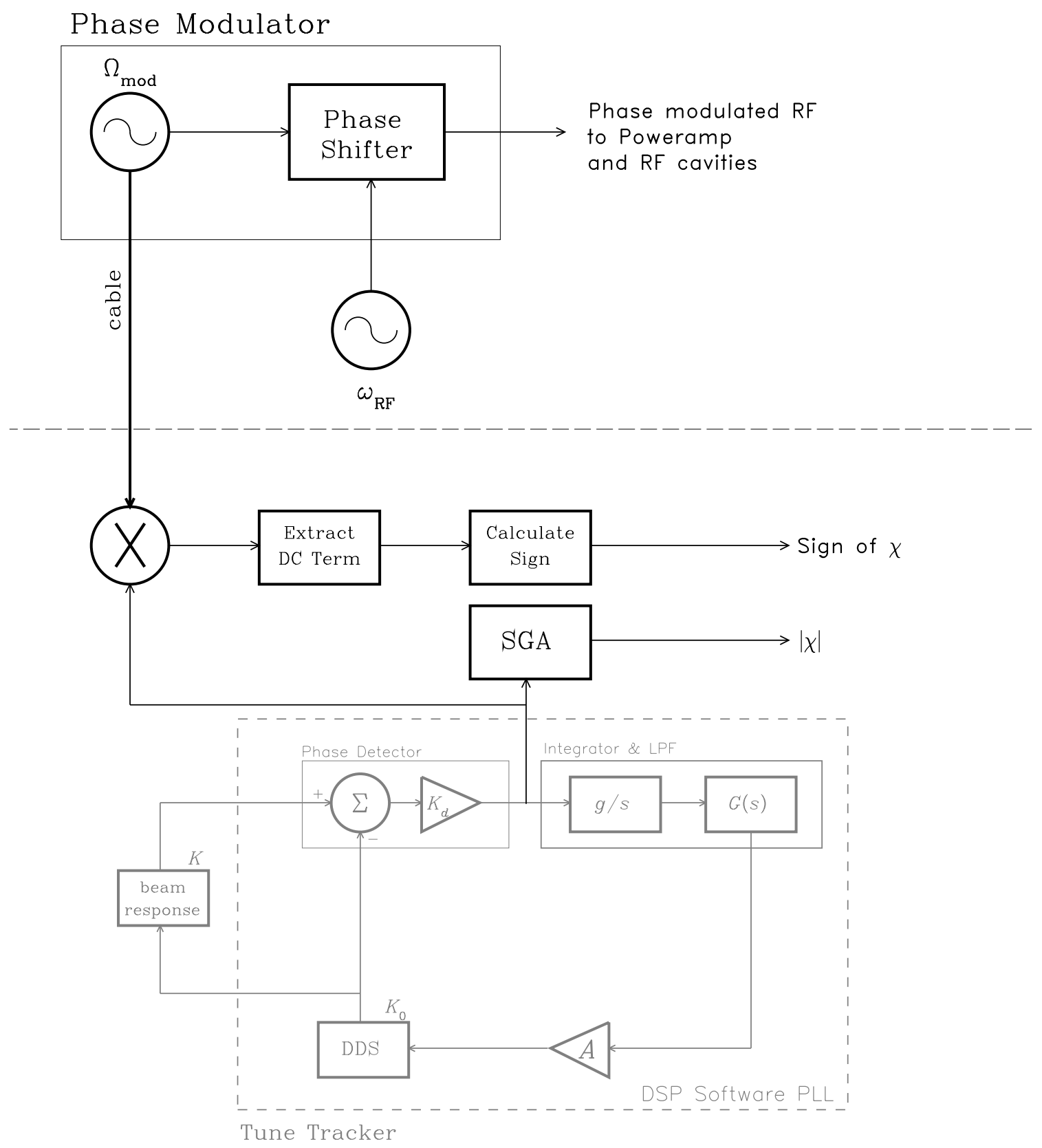

Figure 14 The simplest possible implementation of the CT consists of (a) the addition of a narrow band filter called the Sliding Goertzel Algorithm (SGA) to the output of the TT phase detector, (b) the sign evaluation circuit and (c) the phase modulation circuit to the low level RF system. 


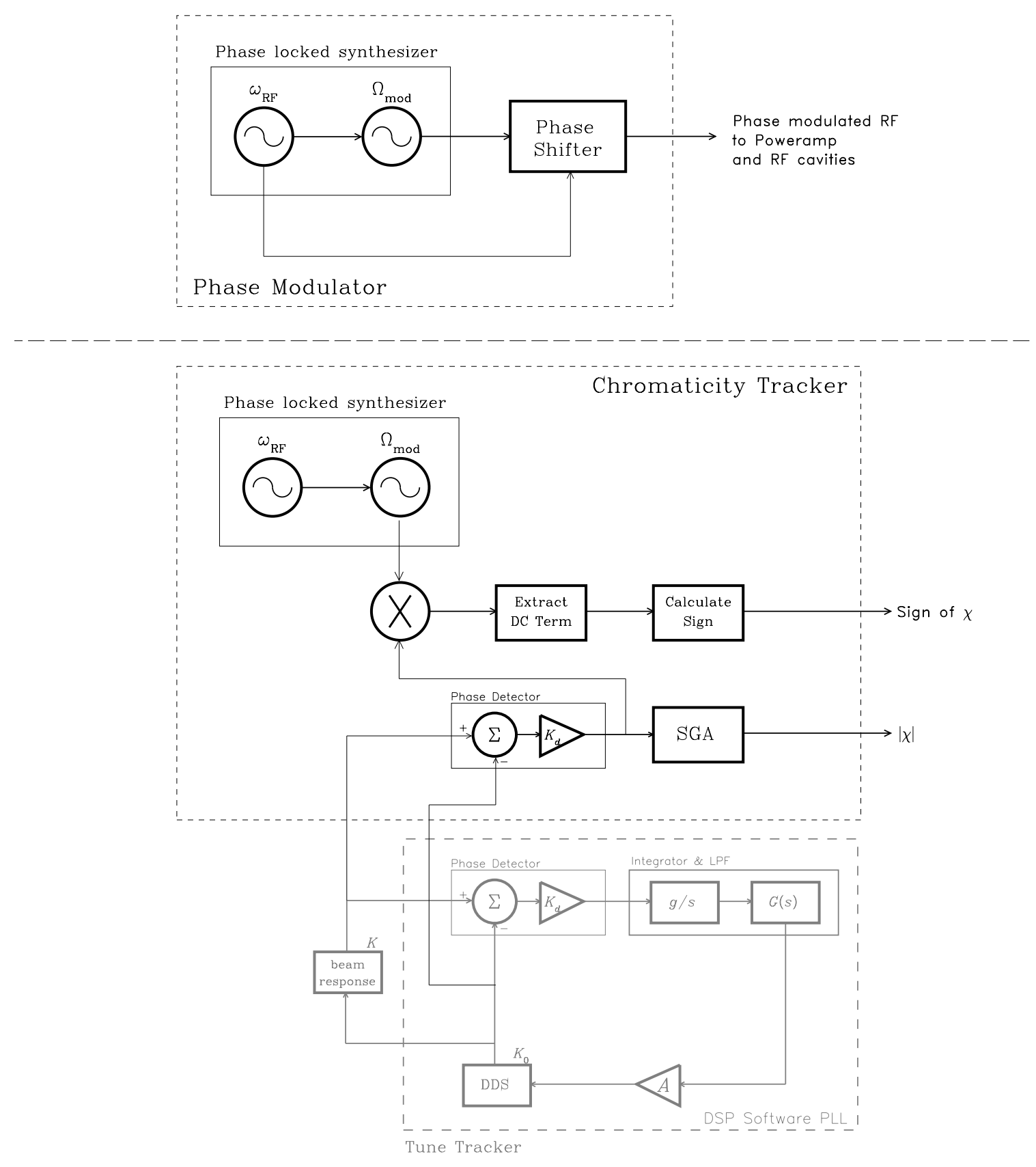

Figure 15 The proposed CT implementation. 
Epilogue 


\section{CONCLUSION}

We have performed a preliminary study of the McGinnis' method and have found that the TT does ignore phase modulations outside its closed loop bandwidth while locked to the betatron tune. We have also verified that a $\Omega_{\bmod }=(2 \pi \times 23) \mathrm{s}^{-1}, \Delta \phi_{\bmod }=5^{\circ}$ phase modulation does not do any harm to the beam and is sufficient for the VSA to measure it. The studies with the $21.4 \mathrm{MHz}$ Schottky and the 3D-BBQ have shown that the chromaticity measured with McGinnis' method is close to what we expect from measuring the chromaticity at one point with the RF change method (See Introduction) and then extrapolating it with a "calibrated" knob. With the qualified success of the McGinnis" method, we have proposed and discussed a design of a CT. The electronics for this design is being drawn up and built at this time.

In the future, after the shutdown, we will have to verify that this method can track the chromaticity up the ramp and through the squeeze. More importantly we will need to show that the beam can survive the phase modulations during this time. Due to time limitations and equipment failure of the head-tail machinery, we were unable to cross check McGinnis's method with the head-tail method. Clearly this needs to be done. All these plans will be finalized once the March to May 2006 shutdown is complete.

\section{ACKNOWLEDGEMENTS}

The author wishes to thank

(i) V. Ranjbar for proof reading this paper.

(ii) The Tevatron group for supporting these studies.

(iii) USLARP for funding part of these studies. 
Appendices 
We want to understand how the 3D-BBQ affects the McGinnis method and to see whether we need to modify any formulas. The 3D-BBQ is essentially a peak detector with a decay time constant that is adjusted with the resistor and capacitor values shown in Figure 17. In order to obtain an analytic solution, we will make the approximation that the current does not decay away between beam passages, i.e. the 3D-BBQ behaves like a sample and hold circuit. See Figure 16.

We will show that

(i) The folding down of any high frequency modes comes from the turn by turn sampling of the beam.

(ii) The 3D-BBQ enhances the baseband mode by a factor of $\sim T_{\mathrm{rev}} / \sigma$ where $\sigma$ is the bunch length and $T_{\text {rev }}$ is the period of the revolution.

(iii) The formula for calculating the chromaticity is the case when $k=0$.

\section{D-BBQ Approximation}

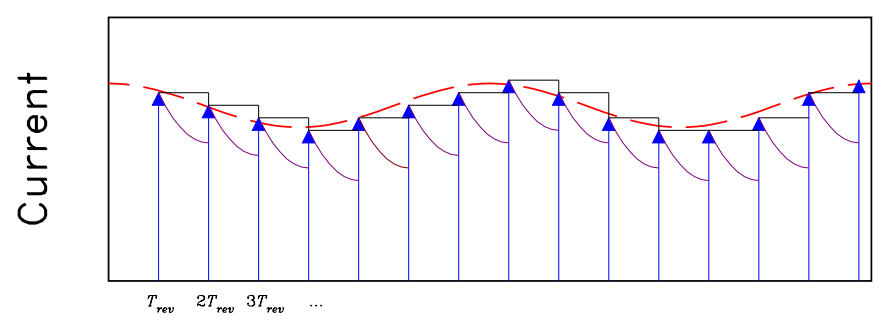

Time

Figure 16 The approximation that we have made to make the 3D$\mathrm{BBQ}$ calculation tractable is to ignore the decay (purple trace) of the sampling between beam passages and replace the decay with constant current (black trace). 
Pickup without 3D-BBQ
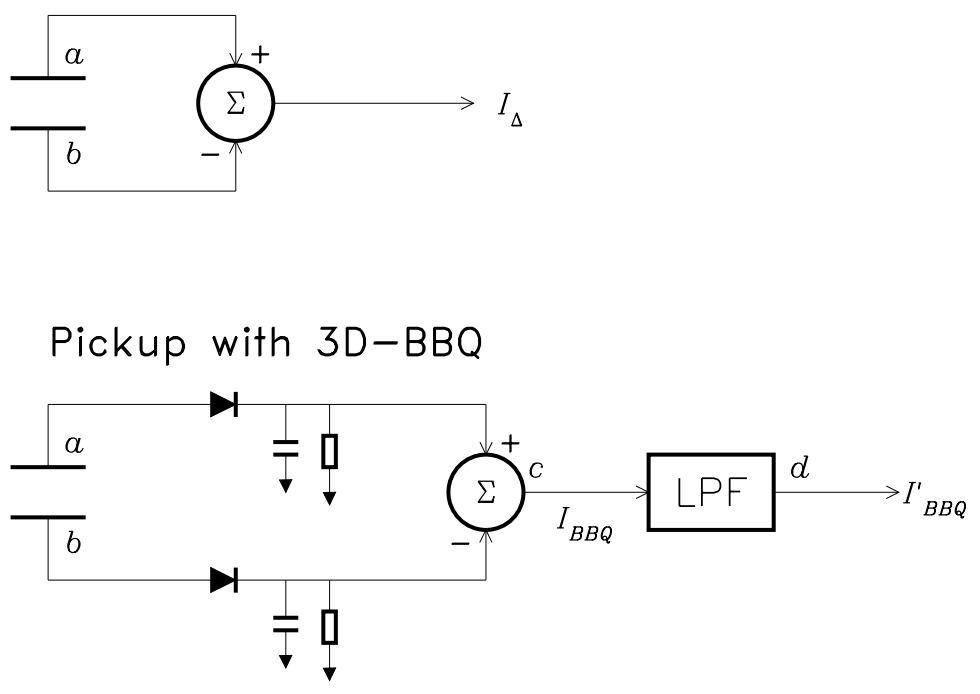

Figure 17 The two setups used in our calculation. The low pass filter (LPF) cuts off all signals above $\omega_{\text {rev }}$.

\section{Pickup with Flat Frequency Response}

We start with the simplest possible situation where we only have a betatron oscillation without any RF phase modulation or synchrotron motion, then looking at Figure 17, the current at point $a, b$ is given by

$$
I_{a, b}(t)= \pm \frac{q_{b} A}{2 \sqrt{\epsilon}} \sum_{k=-\infty}^{\infty} \delta\left(t-k T_{\mathrm{rev}}\right) \cos k \Omega_{0} T_{\mathrm{rev}}+\text { common mode term }
$$

where $T_{\text {rev }}$ is the period of the revolution and the other symbols have already been defined in Theory. Taking the difference between $I_{a}$ and $I_{b}$ and ignoring the common mode term, we have

$$
I_{\Delta}(t)=\frac{q_{b} A}{\sqrt{\epsilon}} \sum_{k=-\infty}^{\infty} \delta\left(t-k T_{\mathrm{rev}}\right) \cos k \Omega_{0} T_{\mathrm{rev}}
$$

Note that this result is identical to (4) if $\delta\left(t-k T_{\mathrm{rev}}\right) \rightarrow \omega_{\mathrm{rev}} \delta\left(\omega_{\mathrm{rev}} t-2 n \pi\right), \Omega_{\mathrm{mod}}=0$, $\Omega_{s}=0$ and $\phi_{r}(0)=0$. 
If we define the Fourier transform as

$$
\tilde{f}(\omega)=\int_{-\infty}^{\infty} d t f(t) e^{-i \omega t}
$$

and use it to Fourier transform (27), we see that

$$
\begin{aligned}
\tilde{I}_{\Delta}(\omega) & =\frac{q_{b} A}{\sqrt{\epsilon}} \sum_{k=-\infty}^{\infty} e^{-i k \omega T_{\mathrm{rev}}} \cos k \Omega_{0} T_{\mathrm{rev}} \\
& =\frac{q_{b} A}{2 \sqrt{\epsilon}} \sum_{k=-\infty}^{\infty} e^{i k\left(\Omega_{0}-\omega\right) T_{\mathrm{rev}}}+e^{-i k\left(\Omega_{0}+\omega\right) T_{\mathrm{rev}}}
\end{aligned}
$$

The infinite sum of exponentials can be written in terms of $\delta$-functions using the Poisson sum formula

$$
\sum_{k=-\infty}^{\infty} e^{i k x}=2 \pi \sum_{k=-\infty}^{\infty} \delta(x-2 k \pi)
$$

Applying this formula, we find that (29) becomes

$$
\tilde{I}_{\Delta}(\omega)=\frac{q_{b} A}{\sqrt{\epsilon}} \times \frac{1}{2 T_{\mathrm{rev}}} \times 2 \pi \sum_{k=-\infty}^{\infty} \delta\left(\omega-\Omega_{0}+k \omega_{\mathrm{rev}}\right)+\delta\left(\omega+\Omega_{0}+k \omega_{\mathrm{rev}}\right)
$$

We can see immediately that in Fourier space, any $\Omega_{0}$ that is sampled periodically $T_{\text {rev }}$ will end up in pairs around $k \omega_{\text {rev. }}$ See Figure 18.

Now when we connect up the 3D-BBQ like in Figure 17. The current at point $c$ is

$$
I_{\mathrm{BBQ}}(t)=\lim _{\sigma \rightarrow 0} \frac{q_{b} A}{\sqrt{\epsilon}} \times \frac{1}{\sqrt{2 \pi} \sigma} \times \sum_{k=-\infty}^{\infty}\left[u\left(t-k T_{\mathrm{rev}}\right)-u\left(t-(k+1) T_{\mathrm{rev}}\right)\right] \cos k \Omega_{0} T_{\mathrm{rev}}
$$

where $u(t)$ is the Heaviside operator. The normalization $1 / \sqrt{2 \pi} \sigma$ appears because

$$
\lim _{\sigma \rightarrow 0} \frac{1}{\sqrt{2 \pi} \sigma} e^{-t^{2} / 2 \sigma^{2}}=\delta(t)
$$

Fourier transforming (33), we get

$$
\begin{aligned}
\tilde{I}_{\mathrm{BBQ}}(\omega)= & \lim _{\sigma \rightarrow 0} \frac{q_{b} A}{\sqrt{\epsilon}} \times \frac{1}{\sqrt{2 \pi} \sigma} \times \frac{\sin \frac{1}{2} \omega T_{\mathrm{rev}}}{\frac{1}{2} \omega} \sum_{k=-\infty}^{\infty} e^{-i \omega\left(k+\frac{1}{2}\right) T_{\mathrm{rev}}} \cos k \Omega_{0} T_{\mathrm{rev}} \\
= & \lim _{\sigma \rightarrow 0} \frac{q_{b} A}{\sqrt{\epsilon}} \times \frac{1}{\sqrt{2 \pi} \sigma} \times \frac{\sin \frac{1}{2} \omega T_{\mathrm{rev}}}{\frac{1}{2} \omega} \times \frac{1}{2} e^{-i \omega T_{\mathrm{rev}} / 2} \times \\
& \sum_{k=-\infty}^{\infty} e^{i\left(\Omega_{0}-\omega\right) k T_{\mathrm{rev}}}+e^{-i\left(\Omega_{0}+\omega\right) k T_{\mathrm{rev}}}
\end{aligned}
$$




\section{Current from Pickup}

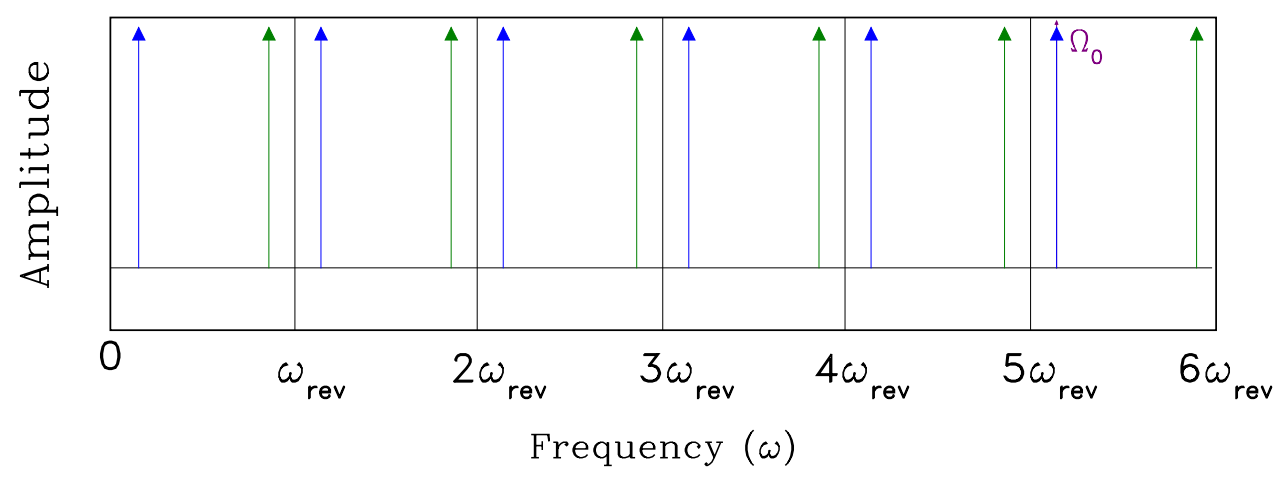

Current After 3D-BBQ Detector

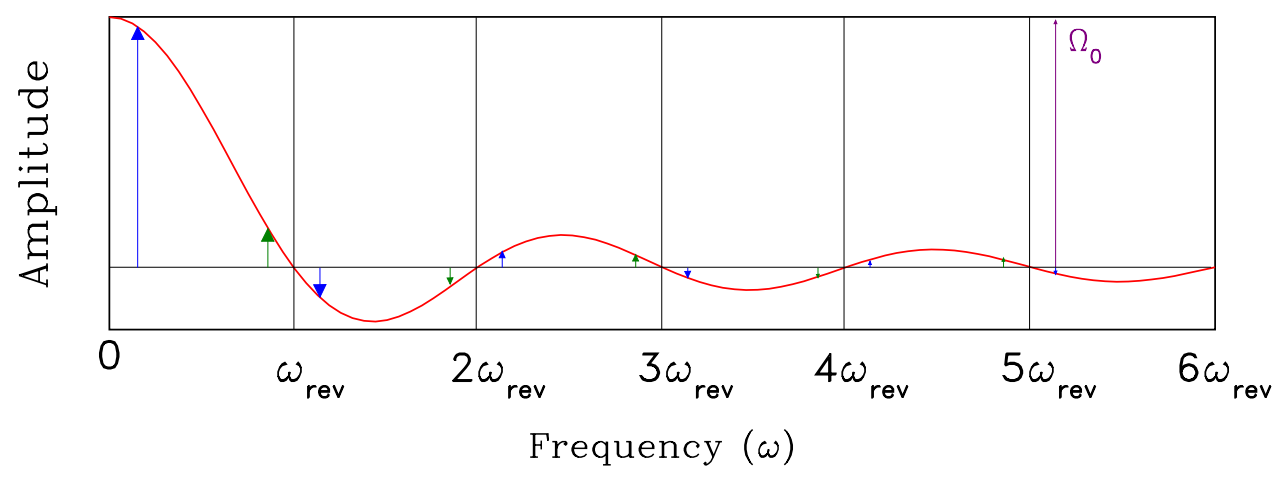

Figure 18 The signal $\Omega_{0}$ is folded down to baseband and repeated in pairs around every $\omega_{\text {rev }}$. In this example $\Omega_{0}=5 \omega_{\text {rev }}+\Delta \Omega$ and so the pairs are $\pm \Delta \Omega$ around $k \omega_{\text {rev }}$.

We apply the Poisson sum formula again to get

$$
\left.\begin{array}{rl}
\tilde{I}_{\mathrm{BBQ}}(\omega)= & \lim _{\sigma \rightarrow 0} \frac{q_{b} A}{\sqrt{\epsilon}} \times \frac{1}{\sqrt{2 \pi} \sigma} \times \frac{\sin \frac{1}{2} \omega T_{\mathrm{rev}}}{\frac{1}{2} \omega T_{\mathrm{rev}}} \times \frac{1}{2} e^{-i \omega T_{\mathrm{rev}} / 2} \times \\
& 2 \pi \sum_{k=-\infty}^{\infty} \delta\left(\omega-\Omega_{0}+k \omega_{\mathrm{rev}}\right)+\delta\left(\omega+\Omega_{0}+k \omega_{\mathrm{rev}}\right)
\end{array}\right\}
$$


Finally, we can express $\tilde{I}_{\mathrm{BBQ}}$ in terms of $\tilde{I}_{\Delta}$

$$
\begin{aligned}
\tilde{I}_{\mathrm{BBQ}}= & \lim _{\sigma \rightarrow 0} \frac{\sin \frac{1}{2} \omega T_{\mathrm{rev}}}{\frac{1}{2} \omega T_{\mathrm{rev}}} \times e^{-i \omega T_{\mathrm{rev}} / 2} \times \frac{1}{\sqrt{2 \pi}}\left(\frac{T_{\mathrm{rev}}}{\sigma}\right) \times \tilde{I}_{\Delta}(\omega) \\
= & (\text { rectangular sampling }) \times\left(\text { constant delay of } T_{\mathrm{rev}} / 2\right) \times \\
& \left(\text { enhancement of baseband signal by } \sim T_{\mathrm{rev}} / \sigma\right) \times \tilde{I}_{\Delta}(\omega) \\
\equiv & \mathcal{M}(\omega) \tilde{I}_{\Delta}(\omega)
\end{aligned}
$$

Thus, this result $\tilde{I}_{\mathrm{BBQ}}$ shows that the baseband $k=0$ mode has been amplified by $\sim T_{\text {rev }} / \sigma$ and this can be quite substantial because $\sigma \ll T_{\text {rev }}$.

\section{Modulations}

With the phase modulation $\Omega_{\bmod }$ and synchrotron modulation $\Omega_{s}$, the current without the 3D-BBQ comes from (4)

$$
\begin{aligned}
\tilde{I}_{\Delta}\left(\omega ; \Omega_{\mathrm{mod}}, \Omega_{s}\right)= & \frac{\omega_{\mathrm{rev}} q_{b}}{4} \frac{A}{\sqrt{\epsilon}} \sum_{k=0}^{\infty} C_{k} \times \\
& \left\{\begin{array}{c}
e^{i \psi_{+}} \sum_{m, n=-\infty}^{\infty} J_{m}\left(Y_{+}\right) J_{n}\left(Z_{+}\right)\left[\delta\left(\omega_{k m n}^{+}-\omega\right)+\delta\left(\omega_{k m n}^{+}+\omega\right)\right] \\
\left.\quad+e^{i \psi_{-}} \sum_{m, n=-\infty}^{\infty} J_{m}\left(Y_{-}\right) J_{n}\left(Z_{-}\right)\left[\delta\left(\omega_{k m n}^{-}-\omega\right)+\delta\left(\omega_{k m n}^{-}+\omega\right)\right]\right\}
\end{array}\right.
\end{aligned}
$$

To obtain $\tilde{I}_{\mathrm{BBQ}}$, we notice from (36) that every line $\delta\left(\omega \pm \Omega_{0}+k \omega_{\text {rev }}\right)$ of $\tilde{I}_{\Delta}$ is constant in size and is multiplied by the transfer function $\mathcal{M}(\omega)$ to obtain $\tilde{I}_{\mathrm{BBQ}}$. Thus we can do exactly the same thing by isolating each mode $(k, m, n, \pm)$ in (37) and applying superposition to sum all the modes if we make the approximation that the 3D-BBQ samples at a fixed period $T_{\text {rev }}$. In fact, the result will look exactly like (36) with $\tilde{I}_{\Delta}(\omega)$ coming from (37).

Referring back to Figure 17, at point $d$, after going through the low pass filter, we need 
only consider $k=0$ term. Thus

$$
\begin{aligned}
& \tilde{I}_{\mathrm{BBQ}}^{\prime}(\omega ; k=0)=\lim _{\sigma \rightarrow 0} \frac{\sin \frac{1}{2} \omega T_{\mathrm{rev}}}{\frac{1}{2} \omega T_{\mathrm{rev}}} \times e^{-i \omega T_{\mathrm{rev}} / 2} \times \frac{1}{\sqrt{2 \pi}}\left(\frac{T_{\mathrm{rev}}}{\sigma}\right) \times \frac{\omega_{\mathrm{rev}} q_{b} A}{4 \sqrt{\epsilon}} \times \frac{1}{2 \pi} \times \\
&\left\{e^{i \psi_{+}} \sum_{m, n=-\infty}^{\infty} J_{m}\left(Y_{+}\right) J_{n}\left(Z_{+}\right)\left[\delta\left(\omega_{0 m n}^{+}-\omega\right)+\delta\left(\omega_{0 m n}^{+}+\omega\right)\right]\right. \\
&+\left.+e^{i \psi_{-}} \sum_{m, n=-\infty}^{\infty} J_{m}\left(Y_{-}\right) J_{n}\left(Z_{-}\right)\left[\delta\left(\omega_{0 m n}^{-}-\omega\right)+\delta\left(\omega_{0 m n}^{-}+\omega\right)\right]\right\}
\end{aligned}
$$

If the TT locks to mode $(k=0, m,+)$, we have

$$
\begin{aligned}
\tilde{I}_{\mathrm{BBQ}}^{\prime}(\omega ; k=0, m,+)= & \lim _{\sigma \rightarrow 0} \frac{\sin \frac{1}{2} \omega T_{\mathrm{rev}}}{\frac{1}{2} \omega T_{\mathrm{rev}}} \times e^{-i \omega T_{\mathrm{rev}} / 2} \times \frac{1}{\sqrt{2 \pi}}\left(\frac{T_{\mathrm{rev}}}{\sigma}\right) \times \frac{\omega_{\mathrm{rev}} q_{b} A}{4 \sqrt{\epsilon}} \times \\
& e^{i \psi_{+}} J_{m}\left(Y_{+}\right) \sum_{n=-\infty}^{\infty} J_{n}\left(Z_{+}\right)\left[\delta\left(\omega_{0 m n}^{+}-\omega\right)+\delta\left(\omega_{0 m n}^{+}+\omega\right)\right]
\end{aligned}
$$

Amazingly, the solution of (39) in temporal space is the $k=0$ mode of

$$
\begin{aligned}
I_{\mathrm{BBQ}}(t ; k=0, m,+) \sim & \sum_{\ell=-\infty}^{\infty}\left[u\left(t-\ell T_{\mathrm{rev}}\right)-u\left(t-(\ell+1) T_{\mathrm{rev}}\right)\right] \times \\
& \cos \left[\omega_{0 m}^{+} \ell T_{\mathrm{rev}}+Z_{+} \sin \left(\Omega_{\mathrm{mod}} \ell T_{\mathrm{rev}}\right)+\psi_{+}\right]
\end{aligned}
$$

where $\omega_{k m}^{ \pm}=\left(k \pm Q_{0}\right) \omega_{\text {rev }}+m \Omega_{s}$. Again, by phase demodulating $I_{\mathrm{BBQ}}(t ; k=0, m,+)$, the chromaticity can be recovered.

\section{Stripline Pickup}

We want to know how the 3D-BBQ behaves with a non-flat response pickup because if we just look at the frequency response of a stripline pickup, there is no DC response

$$
|H(\omega)|=|\sin \omega L / c|
$$

where $L$ is the length of the pickup and $c$ is the velocity of light. The first positive frequency peak of $|H(\omega)|$ is when $\omega L / c=\pi / 2$ and for a $1 \mathrm{~m}$ long stripline pickup, this occurs at $75 \mathrm{MHz}$. But we know that the 3D-BBQ does work with this type of pickup. 
The current from the stripline pickup without the $3 \mathrm{D}-\mathrm{BBQ}$ is

$$
I_{\Delta}(t)=\frac{q_{b} A}{\sqrt{\epsilon}} \sum_{k=-\infty}^{\infty}\left[\delta\left(t-k T_{\mathrm{rev}}\right)-\delta\left(t-k T_{\mathrm{rev}}-2 L / c\right)\right] \cos k \Omega_{0} T_{\mathrm{rev}}
$$

However, with the $3 \mathrm{D}-\mathrm{BBQ}$ which only peak detects the positive $\delta$-function and so

$$
I_{\mathrm{BBQ}}(t)=\frac{q_{b} A}{\sigma \sqrt{\epsilon}} \sum_{k=-\infty}^{\infty}\left[u\left(t-k T_{\mathrm{rev}}\right)-u\left(t-(k+1) T_{\mathrm{rev}}\right)\right] \cos k \Omega_{0} T_{\mathrm{rev}}
$$

which is identical to (32) and so there is no difference to the 3D-BBQ between the flat frequency response pickup and the stripline pickup. 


\section{APPENDIX II: THE SLIDING GOERTZEL ALGORITHM}

The Goertzel algorithm ${ }^{9}(\mathrm{GA})$ is a very efficient way for calculating the Fourier coefficient at one point in frequency space and its main application is in telephone tone detection. The GA is well-known and is usually implemented as an infinite impulse response (IIR) filter. For our purposes, we want to use the GA but in a form that is known as a "sliding" algorithm. Sliding means that the GA is applied for a time sequenced data $\left\{x_{0}, x_{1}, \ldots, x_{N-1}\right\}$ and then the sequence is slid to the right $\left\{x_{1}, x_{2}, \ldots, x_{N}\right\}$ to become the input for the next application of the GA. ${ }^{\dagger}$ This sliding to the right continues ad infinitum and so the output is the time evolution of that Fourier coefficient.

We will derive the sliding Goertzel algorithm (SGA) by first rederiving the GA in the usual form. Unfortunately, the GA in the usual form is not suitable for deriving the SGA and thus we will have to derive another form which we will call the modified GA. With the modified GA, we can easily derive the SGA. We will show that the SGA is very efficient for calculating the Fourier coefficient at one point compared to the discrete Fourier transform (DFT) and even the fast Fourier transform (FFT). We can speed the SGA up even further with a careful choice of the sampling frequency.

\section{Goertzel Algorithm}

We start with the definition of the $N$ point DFT of the sequence $\{x(0), x(1), \ldots, x(N-$ $2), x(N-1)\}$

$$
X(k)=\sum_{n=0}^{N-1} x(n) W_{N}^{k n}
$$

where $W_{N}^{k n}=e^{-i 2 \pi k n / N}$. If we multiply the rhs of (44) by $1=e^{+i 2 \pi k}=W_{N}^{-k N}$ where

$\dagger$ Note that sliding to the right need not be one sample, but for our purposes we will only slide to the right by one. 
$k \in \mathbb{Z}$, we see that

$$
X(k)=\sum_{n=0}^{N-1} x(n) W_{N}^{-k(N-n)}
$$

In order to write (45) as a difference equation, we can expand the rhs and see that

$$
\left.\begin{array}{rl}
X(k) & =x(0) W_{N}^{-k N}+x(1) W_{N}^{-k(N-1)}+\ldots+x(N-1) W_{N}^{-k} \\
& \left.=\left[\ldots\left[\left[x(0) W_{N}^{-k}+x(1)\right] W_{N}^{-k}+x(2)\right] W_{N}^{-k}+\ldots\right] W_{N}^{-k}+x(N-1)\right] W_{N}^{-k}
\end{array}\right\}
$$

Thus we can define $y_{k}(n)$ as

$$
y_{k}(n)=y_{k}(n-1) W_{N}^{-k}+x(n)
$$

with $y_{k}(-1)=0$. Therefore, it is clear from (46) that

$$
X(k)=y_{k}(N) \quad \text { only if } x(N)=0 .
$$

$\ddagger$ The DFT transfer function $H_{\mathrm{DFT}}(z)$ can be easily read off from $(47)$

$$
H_{\mathrm{DFT}}(z)=\frac{1}{1-W_{N}^{-k} z^{-1}}
$$

The GA transfer function $H_{G}(z)$ is obtained by multiplying (49) with

$$
1=\frac{1-W_{N}^{k} z^{-1}}{1-W_{N}^{k} z^{-1}}
$$

so that

$$
H_{G}(z)=\frac{1-W_{N}^{k} z^{-1}}{1-2 \cos (2 \pi k / N) z^{-1}+z^{-2}}
$$

which is a very efficient way of calculating the Fourier coefficient at one point if the direct form II of the filter is used. The direct form of the filter which comes straight from (51) is shown in Figure 19(a). By changing the order of the filters $F_{1}$ and $F_{2}$, it becomes the

$\ddagger$ The statement "if $x(N)=0$ " is critical and is nearly always missing in textbooks ${ }^{10}$ because clearly $X(k) \neq y_{k}(N)$ if this condition is omitted. 
direct form II realization. See Figure 19(b). This gets us the following difference equations which are found in all the literature

$$
\left.\begin{array}{l}
v_{k}(n)=2 \cos (2 \pi k / N) v_{k}(n-1)-v_{k}(n-2)+x(n) \\
y_{k}(n)=v_{k}(n)-W_{N}^{k} v_{k}(n-1)
\end{array}\right\}
$$

with $v(n)=0$ for $n<0$. The efficiency of the direct form II comes from calculating $N$ times the completely real recursive relationship $v(n)$ and then evaluating the complex value $y_{k}(N)=X(k)$ only once at the end.

\section{Modified Goertzel Algorithm}

Unfortunately, the condition "only if $x(N)=0$ " in (48) is an annoyance for deriving the SGA since it is clear that the sequence $\{\ldots, 0, x(0), x(1), x(2), \ldots, x(N-1), x(N), x(N+$ $1), \ldots\}, x(n)$ is not necessarily zero for $n \geq 0$. So instead of using the $y_{k}(n)$ from $(47)$, we define the following

$$
y_{k}^{\prime}(n)=\left[y_{k}^{\prime}(n-1) W_{N}^{-k}+x(n)\right] W_{N}^{-k}
$$

with $y_{k}^{\prime}(-1)=0$ which allows us to get out of our conundrum

$$
X(k)=y_{k}^{\prime}(N-1)=\left[y_{k}^{\prime}(N-2) W_{N}^{-k}+x(N-1)\right] W_{N}^{-k}
$$

which does not have the $x(N)=0$ condition. The modified DFT transfer function $H_{\mathrm{DFT}}^{\prime}(z)$ is read off from (53)

$$
H_{\mathrm{DFT}}^{\prime}(z)=\frac{W_{N}^{-k}}{1-W_{N}^{-k} z^{-1}}=W_{N}^{-k} H_{\mathrm{DFT}}(z)
$$

The modified GA transfer function $H_{G}^{\prime}(z)$ is obtained by multiplying (50) by (55)

$$
H_{G}^{\prime}(z)=\frac{W_{N}^{-k}\left(1-W_{N}^{k} z^{-1}\right)}{1-2 \cos (2 \pi k / N) z^{-1}+z^{-2}}=W_{N}^{-k} H_{G}(z)
$$


(a) Direct Form

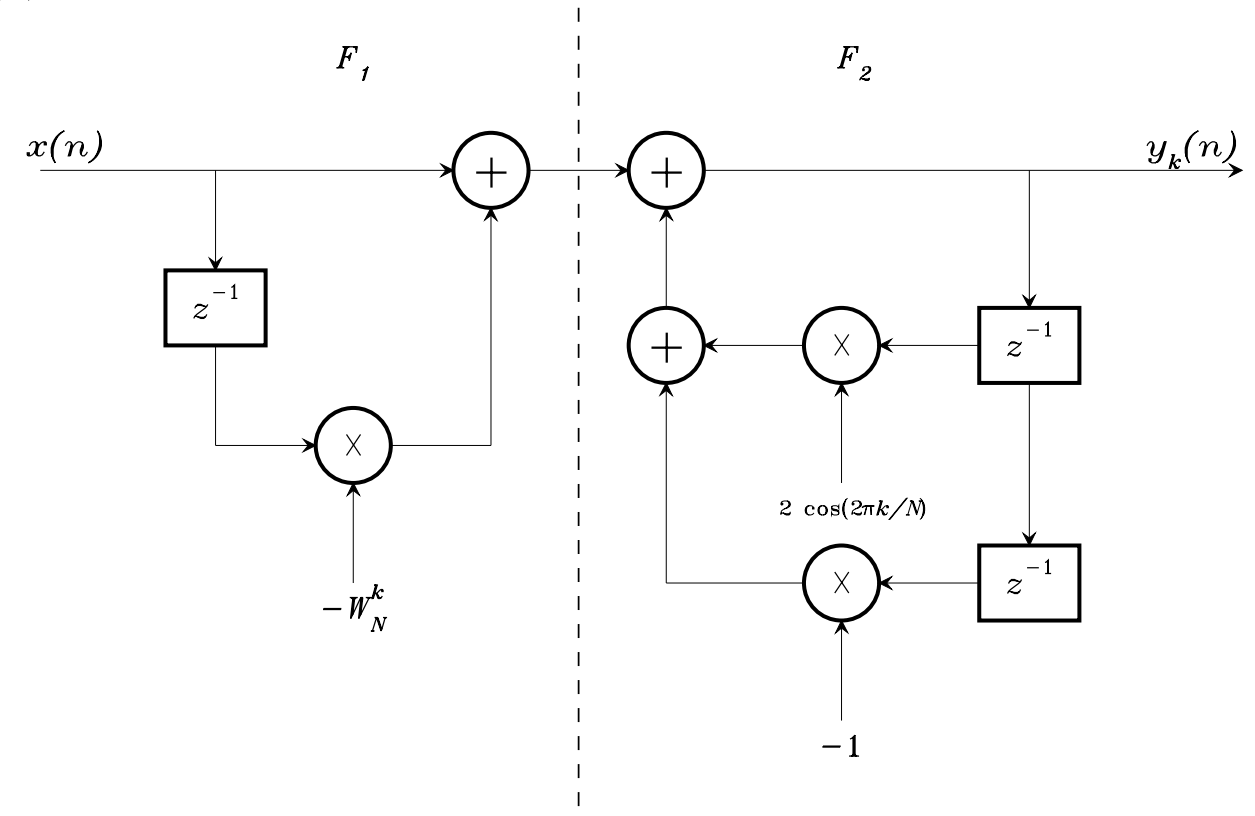

(b) Direct Form II

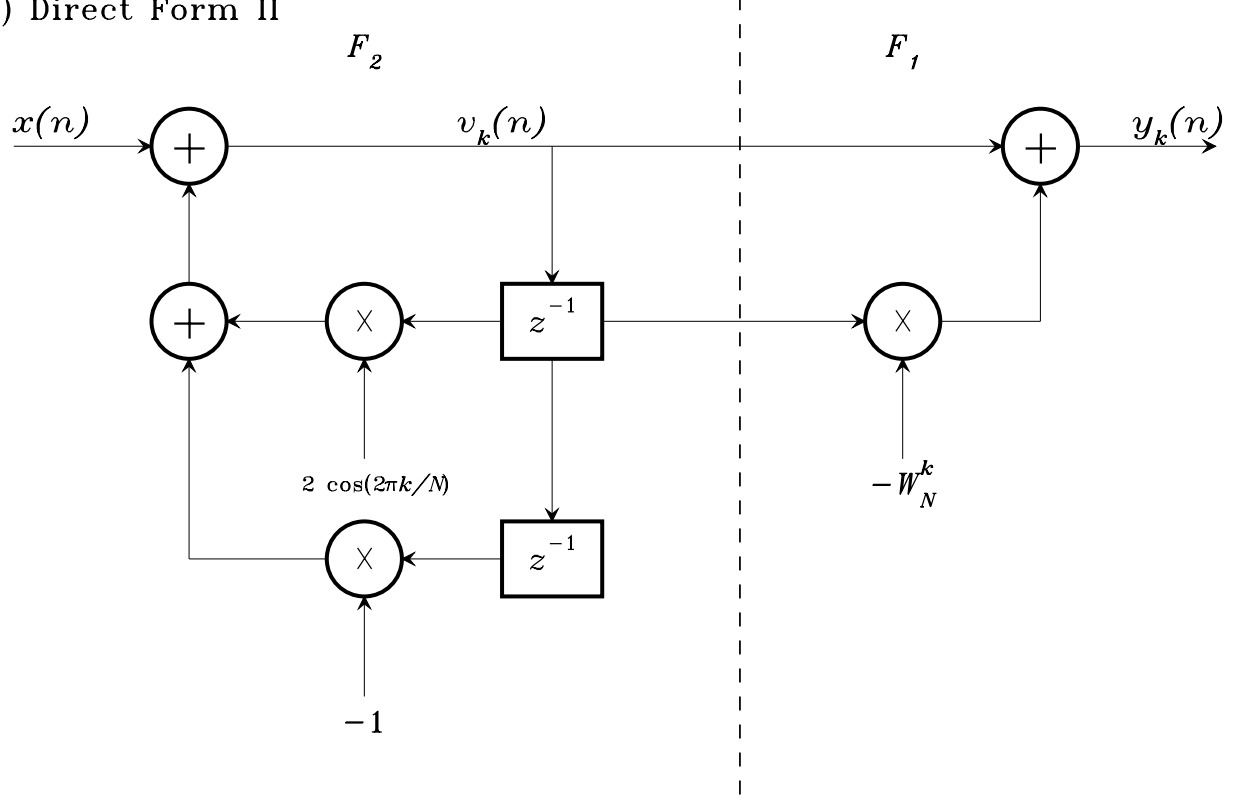

Figure 19 (a) The direct form of $H_{G}$ is shown. (b) Direct form II is the efficient implementation of the GA. The filter $F_{1}$ is only evaluated once for calculating $y_{k}(N)=X(k)$ with $x(N)=0$. 
Thus by putting in the multiplicative constant $W_{N}^{-k}$ to the direct form II implementation of the GA shown in Figure 19(b) we are liberated from the condition $x(N)=0$ for calculating $X(k)$. See Figure 20. From here, the difference equations are

$$
\left.\begin{array}{l}
v_{k}(n)=2 \cos (2 \pi k / N) v_{k}(n-1)-v_{k}(n-2)+x(n) \\
y_{k}^{\prime}(n)=W_{N}^{-k} v_{k}(n)-v_{k}(n-1)
\end{array}\right\}
$$

\section{Modified Goertzel Algorithm}

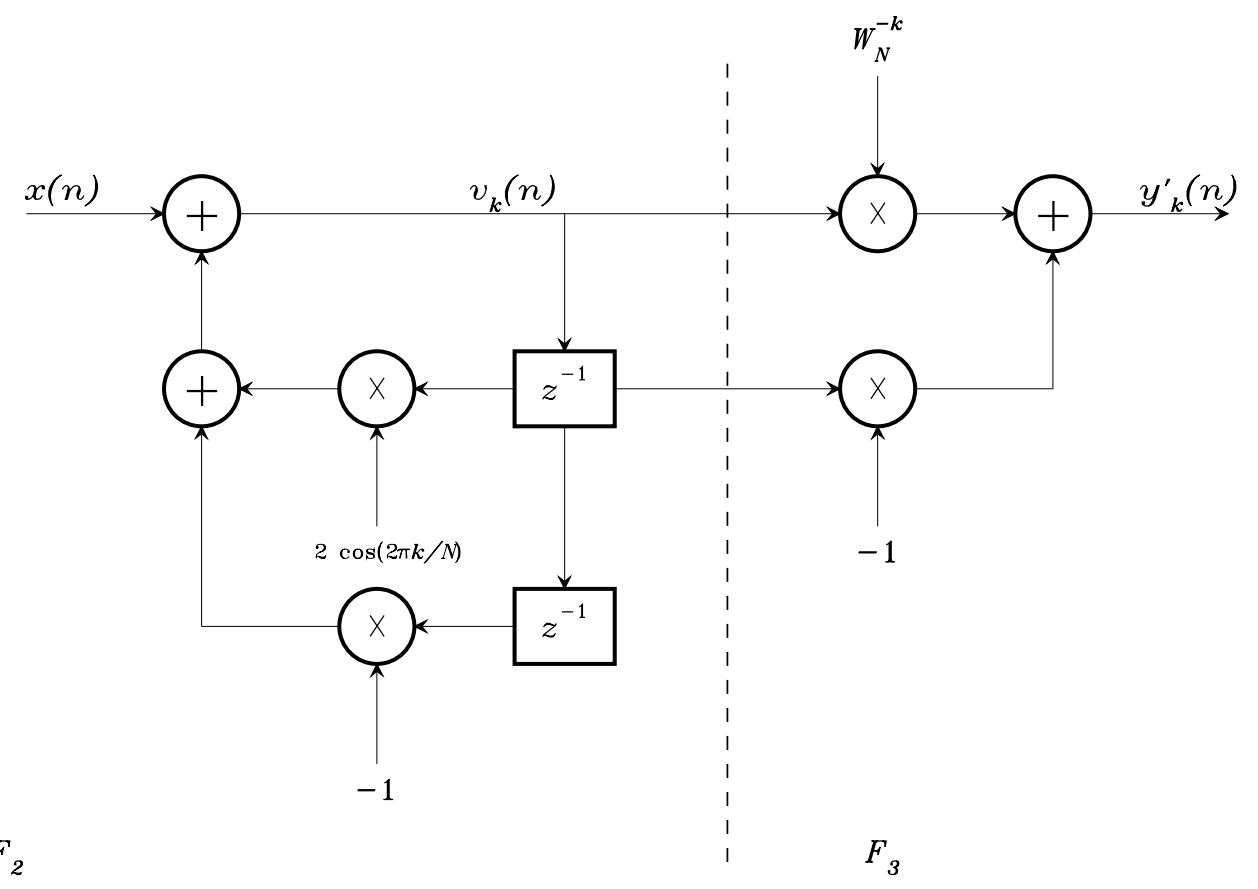

Figure 20 The implementation of the modified GA. Again similar to the GA, the filter $F_{3}$ is only evaluated once for calculating $y_{k}^{\prime}(N-$ 1) $=X(k)$. 


\section{Sliding Goertzel Algorithm}

We have to go back to (44) and introduce the notation

$$
X_{m}(k)=\sum_{n=0}^{N-1} x(m+n) W_{N}^{k n}
$$

which is the DFT of the sequence which starts at $x(m)$. For example,

$$
X_{0}(k)=\sum_{n=0}^{N-1} x(n) W_{N}^{k n}
$$

is the DFT of the sequence $\{x(0), x(1), \ldots, x(N-1)\}$ and

$$
X_{1}(k)=\sum_{n=0}^{N-1} x(n+1) W_{N}^{k n}
$$

is the DFT of the sequence $\{x(1), x(2), \ldots, x(N)\}$. It is easy to show that $X_{m}(k)$ is related to $X_{m-1}(k)$ by the following expression

$$
X_{m}(k)=\left[X_{m-1}(k)-x(m-1)+x(m-1+N)\right] W_{N}^{-k}
$$

Unfortunately, this is not quite useful for deriving the difference equation. We need to do a little bit more work where we renumber the $x()$ 's: $x(0) \rightarrow x(-N+1), x(1) \rightarrow x(-N+2)$, $\ldots, x(N-1) \rightarrow x(0), x(N) \rightarrow x(1), \ldots$. See Figure 21. So (60) becomes

$$
X_{m}(k)=\left[X_{m-1}(k)-x(m-N)+x(m)\right] W_{N}^{-k}
$$

which is a difference equation in $X_{m}$.

Thus the sliding DFT transfer function $H_{\mathrm{SDFT}}(z)$ is

$$
H_{\mathrm{SDFT}}(z)=\frac{W_{N}^{-k}\left(1-z^{-N}\right)}{1-W_{N}^{-k} z^{-1}}
$$

Using the same trick as before, we multiply (62) by (50) to obtain the SGA transfer function $H_{\mathrm{SG}}(z)$

$$
H_{\mathrm{SGA}}(z)=\frac{\left(1-z^{-N}\right) W_{N}^{-k}\left(1-W_{N}^{k} z^{-1}\right)}{1-2 \cos (2 \pi k / N) z^{-1}+z^{-2}}=\left(1-z^{-N}\right) H_{\mathrm{G}}^{\prime}(z)
$$




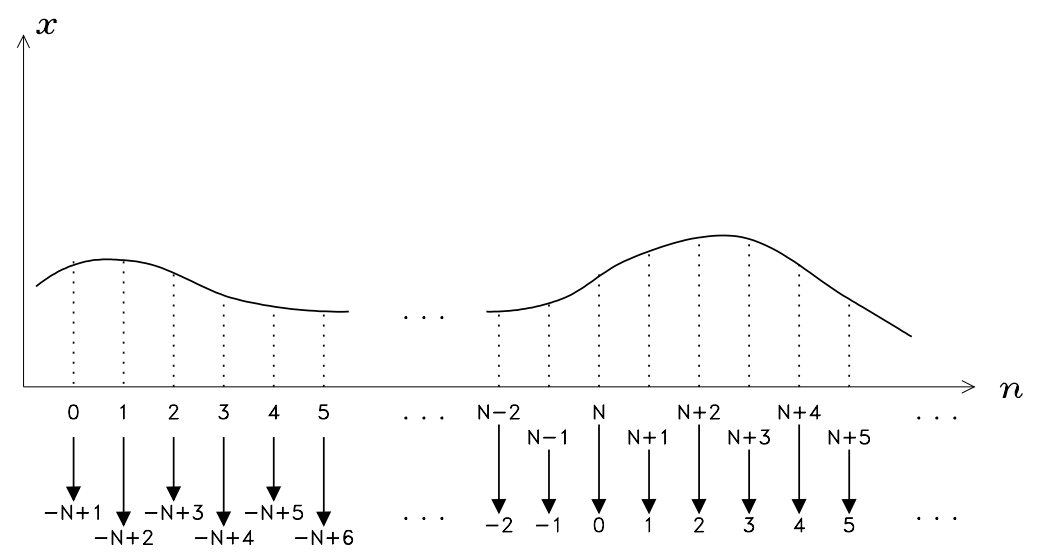

Figure 21 The numbering of the input sequence $x(n)$ is renumbered as shown here.

Finally, the SGA implementation is shown in Figure 22 and the difference equations are

$$
\left.\begin{array}{rl}
v_{k}(n) & =2 \cos (2 \pi k / N) v_{k}(n-1)-v_{k}(n-2)+x(n)-x(n-N) \\
X_{n}(k) & =W_{N}^{-k} v_{k}(n)-v_{k}(n-1)
\end{array}\right\}
$$

Note that because of the renumbering, $v_{k}(n)=0$ and $x(n)=0$ for $n \leq-N$.

\section{Example}

For this example, let the input sequence be $\left\{\ldots, 0,0, x_{0}, x_{1}, x_{2}, x_{3}, x_{4}, \ldots\right\}$. The $N=$ 4 point DFT of the sequence $\left\{x_{0}, x_{1}, x_{2}, x_{3}\right\}$ is

$$
X(k)=x_{0}+x_{1} W+x_{2} W^{2}+x_{3} W^{3}
$$

where $W \equiv W_{4}^{k}$. We will first look at the direct form II GA from Figure 19(b) and the 


\section{Sliding Goertzel Algorithm}

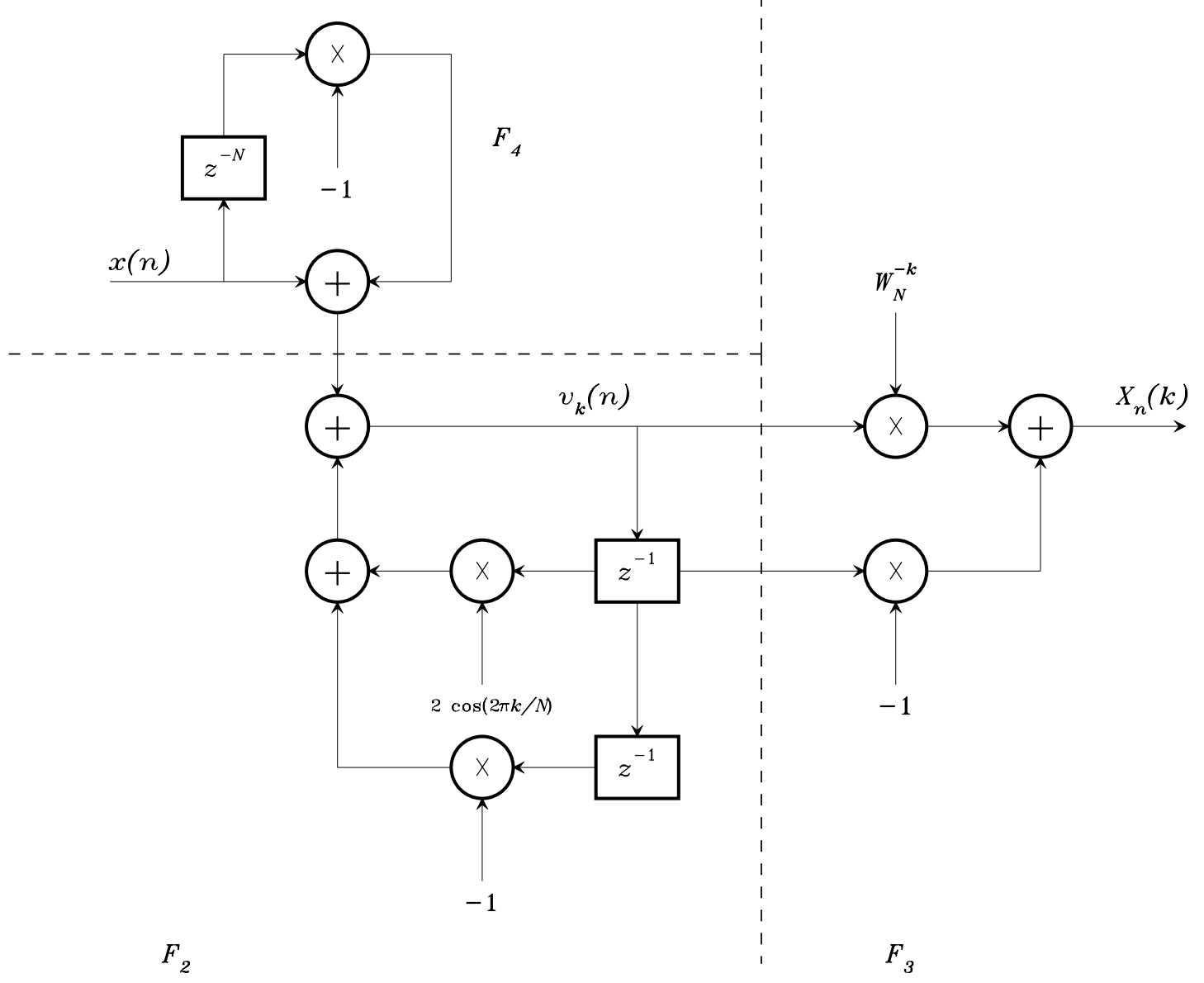

Figure 22 The implementation of the SGA is with the addition of $F_{4}$ to the modified GA shown in Figure 20.

difference equation from (52), we have

$$
\left.\begin{array}{l}
v_{k}(0)=x_{0} \\
v_{k}(1)=a v(0)+x_{1}=a x_{0}+x_{1} \\
v_{k}(2)=\left(a^{2}-1\right) x_{0}+a x_{1}+x_{2} \\
v_{k}(3)=\left(a^{3}-2 a\right) x_{0}+\left(a^{2}-1\right) x_{1}+a x_{2}+x_{3} \\
v_{k}(4)=\left(a^{4}-3 a^{2}+1\right) x_{0}+\left(a^{3}-2 a\right) x_{1}+\left(a^{2}-1\right) x_{2}+a x_{3}+x(4)
\end{array}\right\}
$$


where $v(n)=0$ for $n<0, a=2 \cos (2 \pi k / N)$ and $x(4)=0$. Therefore

$$
\begin{aligned}
y_{k}(4)= & \left(a^{4}-3 a^{2}+1\right) x_{0}+\left(a^{3}-2 a\right) x_{1}+\left(a^{2}-1\right) x_{2}+a x_{3}- \\
& W\left[\left(a^{3}-2 a\right) x_{0}+\left(a^{2}-1\right) x_{1}+a x_{2}+x_{3}\right] \\
= & x_{0}+x_{1} W+x_{2} W^{2}+x_{3} W^{3} \\
= & X(k)
\end{aligned}
$$

where we have used $a=W^{-1}+W$ and the cyclic nature of $W$, i.e. $W^{4}=1$.

For the modified GA using Figure 20, we calculate the $v_{k}$ 's like the above, and calculate $y_{k}^{\prime}(3)$ once at the end to get

$$
\begin{aligned}
y_{k}^{\prime}(3) & =W^{-1} v_{k}(3)-v_{k}(2) \\
& =W^{-1}\left[\left(a^{3}-2 a\right) x_{0}+\left(a^{2}-1\right) x_{1}+a x_{2}+x_{3}\right]-\left(a^{2}-1\right) x_{0}+a x_{1}+x_{2} \\
& =x_{0}+x_{1} W+x_{2} W^{2}+x_{3} W^{3} \\
& =X(k)
\end{aligned}
$$

as required.

Finally, for the SGA, we have to renumber the input sequence: $x_{0} \rightarrow x(-3), x_{1} \rightarrow$ $x(-2), x_{2} \rightarrow x(-1), x_{3} \rightarrow x(0), x_{4} \rightarrow x(1)$, etc. and so

$$
\begin{aligned}
v_{k}(-3) & =x(-3)=x_{0} \\
v_{k}(-2) & =a v_{k}(-3)+x(-2)=a x_{0}+x_{1} \\
v_{k}(-1) & =\left(a^{2}-1\right) x_{0}+a x_{1}+x_{2} \\
v_{k}(0) & =\left(a^{3}-2 a\right) x_{0}+\left(a^{2}-1\right) x_{1}+a x_{2}+x_{3} \\
v_{k}(1) & =\left(a^{4}-3 a^{2}+1\right) x_{0}+\left(a^{3}-2 a\right) x_{1}+\left(a^{2}-1\right) x_{2}+a x_{3}+x_{4}
\end{aligned}
$$

where $x(n)=0$ and $v_{k}(n)=0$ for $n \leq-4$.

$$
\left.\begin{array}{rl}
X_{0}(k) & =W^{-1}\left[\left(a^{3}-2 a\right) x_{0}+\left(a^{2}-1\right) x_{1}+a x_{2}+x_{3}\right]-\left(a^{2}-1\right) x_{0}-a x_{1}-x_{2} \\
& =x_{0}+x_{1} W+x_{2} W^{2}+x_{3} W^{3} \\
& =X(k)
\end{array}\right\}
$$


and sliding over to the next sequence $\left\{x_{1}, x_{2}, x_{3}, x_{4}\right\}$, the SGA gives

$$
\left.\begin{array}{rl}
X_{1}(k)= & W^{-1}\left[\left(a^{4}-3 a^{2}+1\right) x_{0}+\left(a^{3}-2 a\right) x_{1}+\left(a^{2}-1\right) x_{2}+a x_{3}+x_{4}\right]- \\
& \left(a^{3}-2 a\right) x_{0}-\left(a^{2}-1\right) x_{1}-a x_{2}-x_{3} \\
= & x_{1}+x_{2} W+x_{3} W^{2}+x_{4} W^{3}
\end{array}\right\}
$$

which is exactly what we expect the DFT to be (and the $x_{0}$ term magically disappears because its coefficient contains $\left.\left(1-W^{4}\right)=0\right)$.

\section{Efficiencies}

The efficiency of the $N$ point DFT, SDFT, GA, modified GA, SGA, and Radix-2 FFT for calculating one point in Fourier space is summarised in Table 3. Clearly, after doing the calculations for the first time, SGA wins hands down because the number of multiplies and additions is constant. In fact, the SGA efficiency can be further improved by a judicious choice of sampling frequency. This will be discussed in the next section.

\begin{tabular}{c|c|c|c|c}
\hline \multicolumn{1}{l}{ Table 3. One Fourier Point from $N$ Real Data Points Efficiencies } \\
\hline & $X_{0}(k)$ & & $X_{1}(k)$ & \\
\hline \multirow{2}{*}{ Algorithm } & \# Multiplies & \# Adds & \# Multiplies & $\#$ Adds \\
\hline DFT & $2 N$ & $2 N$ & $2 N$ & $2 N$ \\
SDFT & $2 N$ & $2 N$ & 4 & 4 \\
GA & $N+2$ & $2 N$ & $N+2$ & $2 N$ \\
modified GA & $N+1$ & $2 N-1$ & $N+1$ & $2 N-1$ \\
SGA & $N+1$ & $2 N-1$ & 3 & 5 \\
special SGA & 0 & $2 N-4$ & 0 & 2 \\
Radix-2 FFT & $\log _{2} N+1$ & $\frac{3}{2} \log _{2} N+\frac{1}{2}$ & $\log _{2} N+1$ & $\frac{3}{2} \log _{2} N+\frac{1}{2}$ \\
\hline \hline
\end{tabular}




\section{Special SGA}

Since the modulation of the betatron tune is at a fixed frequency $f_{m}$, we can in fact

choose the sampling frequency $f_{S}$ so that $\cos (2 \pi k / N)=0 .{ }^{11}$ In particular, $k=N f_{m} / f_{S}$ and thus we require $f_{m} / f_{S}=(2 p+1) / 4$ where $p \in \mathbb{N}$. However, we require $f_{S}>f_{m}$ and so there are only two solutions, i.e. when $p=0$ or 1 . This implies that $f_{S}=4 f_{m}$ or $f_{S}=4 f_{m} / 3$. The Nyquist criterion demands that $f_{S}>2 f_{m}$ and so there can be only one solution

$$
f_{S}=4 f_{m}
$$

The difference equations for this special SGA are

$$
\left.\begin{array}{rl}
v_{k}(n) & =-v_{k}(n-2)+x(n)-x(n-N) \\
X_{n}(k) & =i v_{k}(n)-v_{k}(n-1)
\end{array}\right\}
$$

with $v_{k}(n)=0$ and $x(n)=0$ for $n \leq-N$.

We can immediately see the advantages of the special SGA:

(i) No multiplications. This gives an immediate speed up.

(ii) No round off errors from the cosine term. The SGA filter is on the edge of instability because the poles are on the unit circle in the $z$-transform plane. Any rounding errors can move the poles off the unit circle and thus cause the filter to become unstable.

(iii) $f_{m}$ is in the centre of a frequency bin and so there is no leakage to adjacent bins.

The frequency response of the special SGA for $N=40$ and $N=80$ is shown in Figure 23. 


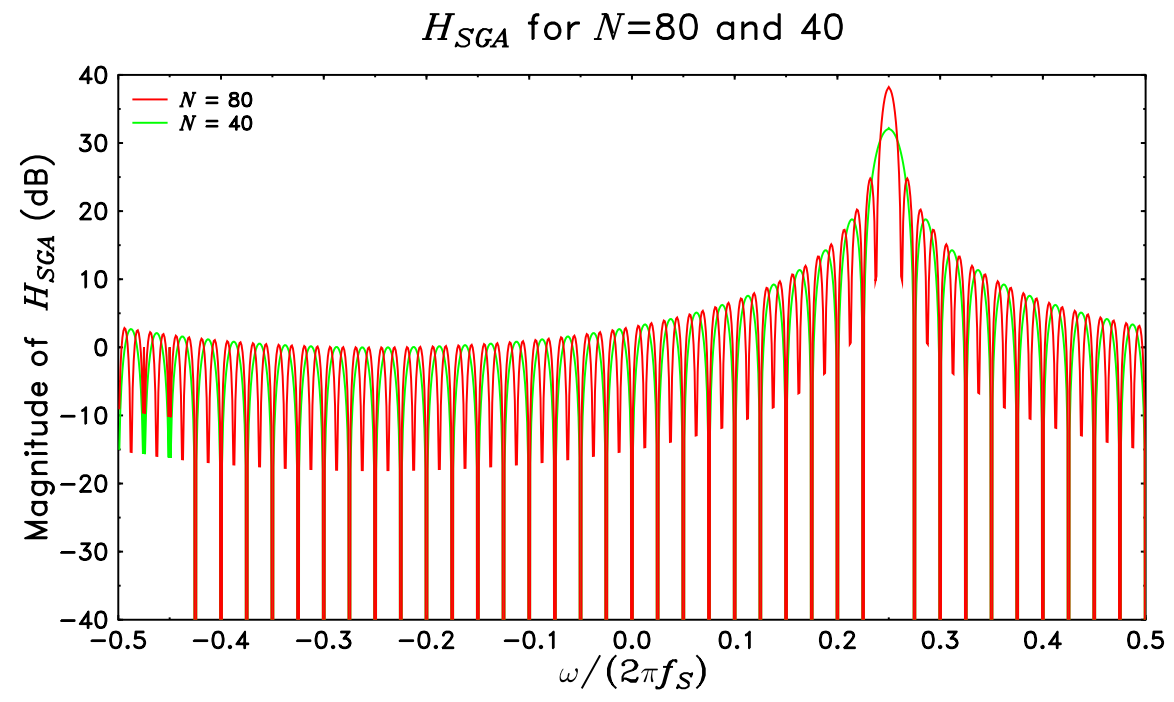

Figure 23 The magnitude response of the special SGA when $N=$ 40 and 80 . Notice that the peak of the response is exactly at $f_{S} / 4$. 


\section{REFERENCES}

[1] D. McGinnis, Chromaticity Measurements using Phase Modulated RF and Vector Signal Analyzers, PBAR Note 656, Fermilab, 2001.

[2] V. Ranjbar, Extracting Chromaticity from Both Planes at the Same Time Using the Head-tail Phase Shift Technique, Beams Document 1330-v1, Fermilab, 2004.

[3] C.Y. Tan, The Tevatron Tune Tracker PLL - Theory, Implementation and Measurements, TM-2275, Fermilab 2004.

[4] C.Y. Tan and V. Ranjbar, Tevatron Electronic Log Book, 12 May 2005.

[5] M. Gasior, Direct Diode Detection (3D) Base-Band Q (BBQ) Measurement, http:// mgasior .home. cern. ch/mgasior/pro/3D-BBQ/3D-BBQ.html.

[6] H. Huang et al, Experimental Determination of the Hamiltonian for Synchrotron Motion with RF Phase Modulation, Phys. Rev. E, 48, 4678 (1993).

[7] S.Y. Lee, Accelerator Physics, 2nd Edition, World Scientific, Singapore, 1999, p. 272.

[8] C.Y. Tan and J. Steimel, The Tevatron Longitudinal Dampers, TM-2184, Fermilab Technical Memo, 2002.

[9] E. Jacobson and R. Lyons, The Sliding DFT, IEEE Signal Processing Magazine, March 2003, p. 74. Note: this reference is for completeness only, there are just too many errors.

[10] J.G. Proakis and D.G. Manolakis, Digital Signal Processing Principles - Algorithms, and Applications 3rd Edition, Prentice Hall, 1996.

[11] E. Kiser, Digital Decoding Simplified - Sequential Exact-Frequency Goertzel Algorithm, Circuit Cellar Ink, 182, 2005, p. 22. 